\title{
Combining Analytical Hierarchy Process and Choquet integral within Non Addititive Robust Ordinal Regression
}

\author{
Salvatore Corrente ${ }^{\mathrm{a}}$, Salvatore Greco $^{\mathrm{a}, \mathrm{b}}$, Alessio Ishizaka $^{\mathrm{b}}$ \\ ${ }^{a}$ Department of Economics and Business, University of Catania, Corso Italia, 55, 95129 Catania, Italy \\ ${ }^{b}$ University of Portsmouth, Portsmouth Business School, Centre of Operations Research and Logistics (CORL), \\ Richmond Building, Portland Street, Portsmouth PO1 3DE, United Kingdom
}

\begin{abstract}
We consider multiple criteria decision aiding in case of interaction between criteria. In this case the usual weighted sum cannot be used to aggregate evaluations on different criteria and other value functions with a more complex formulation have to be considered. The Choquet integral is the most used technique and also the most widespread in the literature. However, the application of the Choquet integral presents two main problems being the necessity to determine the capacity, which is the function that assigns a weight not only to all single criteria but also to all subset of criteria, and the necessity to express on the same scale evaluations on different criteria. While with respect to the first problem we adopt the recently introduced Non Additive Robust Ordinal Regression (NAROR) taking into account all the capacities compatible with the preference information provided by the DM, with respect to the second one we build the common scale for the considered criteria using the Analytic Hierarchy Process (AHP). We propose to use AHP on a set of reference points in the scale of each criterion and to use an interpolation to obtain the other values. This permits to reduce considerably the number of pairwise comparisons usually required by the DM when applying AHP. An illustrative example details the application of the proposed methodology.
\end{abstract}

Keywords: Multiple Criteria Decision Aiding, Interaction between criteria, Choquet integral, Analytical Hierarchy Process, Non Additive Robust Ordinal Regression

\section{Introduction}

In Multiple Criteria Decision Aiding (MCDA) problems (see [42] for an accessible guide to MCDA and [20] for a comprehensive collection of state of the art surveys), a set of alternatives $A=\{a, b, c, \ldots\}$ is evaluated on a set of evaluation criteria $G=\left\{g_{1}, \ldots, g_{n}\right\}$ (sometimes, for the sake of simplicity and slightly abusing of the notation, we refer to the criteria with their indices, i.e. we shall write $i \in G$, instead of $g_{i} \in G$ ). Typical MCDA problems are choice, sorting and ranking. Choice problems consist of choosing a subset (possibly composed of one element only) $A^{*} \subseteq A$ of alternatives considered the best; sorting problems consist of assigning each alternative to one or more predefined and preferentially ordered contiguous classes, while ranking problems consist of partially or completely ordering all alternatives from the best to the worst.

Email addresses: salvatore.corrente@unict.it (Salvatore Corrente), salgreco@unict.it (Salvatore Greco), Alessio.Ishizaka@port.ac.uk (Alessio Ishizaka)

[Post-print] Please cite as: Corrente Salvatore, Greco Salvatore, Ishizaka Alessio, Combining Analytical Hierarchy Process and Choquet integral within Non Addititive Robust Ordinal Regression, OMEGA, online advance publications, 10.1016/j.omega.2015.07.003 
Looking at the evaluations of the alternatives on the criteria, without taking into account further preference information and any preference model, it could be only observed if the dominance relation is fulfilled by some pairs of alternatives ${ }^{2}$. In general, the dominance relation provides really poor information and leaves many alternatives incomparable. For this reason, to get more precise recommendations on the problem at hand, there is the necessity to aggregate the evaluations of the alternatives on the considered criteria through some appropriate preference model representing the preferences of the Decision Maker (DM). In the literature the most well-known aggregation methods are the Multi-Attribute Value Theory (MAVT) [45] and the outranking methods (for ELECTRE methods see $[21,23,54]$ and for PROMETHEE methods see [11, 12]). MAVT assigns to each alternative $a$ a real number $U(a)$ being representative of the degree of desirability of $a$ with respect to the problem at hand, while outranking methods are based on an outranking relation being a binary relation $S$ on the set of alternatives $A$, such that $a S b$ means that $a$ is at least as good as $b$.

Both family of methods are based on the mutual preference independence between criteria [45, 64] but, in many real world decision making problems, the evaluation criteria are not independent but interacting. For instance, suppose the DM likes sport cars and she wants to buy a car taking into account the criteria price, maximum speed and acceleration. In this case, maximum speed and acceleration can be considered negatively interacting criteria while maximum speed and price can be considered positively interacting criteria. In fact, on one hand, even if maximum speed and acceleration are very important for a DM liking sport cars, in general cars with a high maximum speed have also a good acceleration and, therefore, the comprehensive importance of the two criteria considered together should be smaller than the sum of the importance of the two criteria considered alone. On the other hand, a car with a high maximum speed has often also a high price and, therefore, a car with a high maximum speed and a moderate price is very well appreciated. Consequently, the comprehensive importance of these two criteria considered together should be greater than the sum of the importance of the two criteria considered alone.

In such cases, the mutual preference independence can be violated because, for example, due to the positive interaction between maximum speed and price, at a given level of price, one can prefer one combination of maximum speed and acceleration, while at another level of price one can prefer another combination of maximum speed and acceleration. Observe however that the violation of preference independence does not imply that the considered family of criteria is no more consistent. Indeed, consistency [54] refers to the requirements of monotonicity, that is, when improving the evaluations on considered criteria the overall evaluation of an alternative cannot be deteriorated, exhaustivity, that is, all the relevant criteria are considered, and non-redundancy, that is, no criterion can be removed without losing the representation of a relevant point of view. Monotonicity, exhaustivity and non-redundancy can continue to be satisfied also when preference independence does not hold. For instance, in the didactic example of Section 2, we show how reasonable can be the overall evaluations of students obtained aggregating scores in different subjects by the Choquet integral [14] rather than by the weighted sum. If the problem is correctly formulated, aggregation through Choquet integral satisfies monotonicity, exhaustivity and non-redundancy even if, as explained in the example, it does not satisfy preference independence.

Interaction between criteria and violation of the preference independence are well known in MCDA (see e.g. [7, 19, 26, 44]). In the following, we briefly survey several methods handling with the interaction between criteria. Considering the utility functions as aggregation methods, the multilinear utility function [45] and the UTA ${ }^{G M S}$-INT [38] are reported in the literature. The first one aggregates performances on considered criteria through a weighted sum of products of marginal utilities

\footnotetext{
${ }^{2}$ An alternative $a$ dominates an alternative $b$ if the evaluations of $a$ are at least as good as the evaluations of $b$ on all criteria and better for at least one criterion.
} 
corresponding to single criteria, over all subsets of criteria, while UTA ${ }^{G M S}$-INT is based on enriched additive value functions that add some further terms representing interaction between criteria to the usual sum of marginal utility functions. In Artificial Intelligence (AI), interaction between criteria has been recently considered through GAI-networks [30] as well as through UCP-networks [10], that are based on the idea of Generalized Additive Independence (GAI) decomposition [25]. Positive and negative interaction between criteria has been taken into account also in outranking methods such as ELECTRE [24] and PROMETHEE [15]. Another method that takes into consideration interaction between criteria is the Analytical Network Process (ANP) [58]. In this case, interaction between criteria is one of the possible results of interdependencies and network between goals, criteria and alternatives. Observe that very specific interactions between criteria can be considered within ANP. For instance, ANP can model interactions that depend on the considered alternatives. This is the case of a positive interaction between criteria "price" and "maximum speed" for evaluating an economic car, which is not the case for a sport car. Considering interaction between criteria that can change from an alternative to another is not possible with the Choquet integral for which interaction between criteria holds in the same way for all the alternatives. However, the price to pay for such so fine modeling is an increased amount of preference information that can be difficult to supply for the DM.

Even if all cited methods are able to deal with the interaction between criteria, the most well-known methodologies in the literature are the non-additive integrals, such as the Choquet integral (see [14] for the original Choquet integral and [31] for the application of the Choquet integral in MCDA), the Sugeno integral [63] and the generalizations of the Choquet integral, that are the bipolar Choquet integral [34] or the level dependent Choquet integral[36]. The basic idea of these approaches is that the interaction between criteria can be modeled through a capacity, called also fuzzy measure, assigning a weight not only to each criterion but also to each subset of criteria.

In this paper, we shall consider the Choquet integral because, currently, it is the most adopted methodology to deal with interactions between criteria for its manageability (for example, we shall see that we can use linear programming to determine capacities compatible with DM's preferences) and for the meaningfulness of its preference parameters, namely the capacity that becomes understandable and intelligible even for the non expert DM using some specific techniques such as the Möbius representation, the Shapley index and the interaction indices.

Even if it is theoretically appealing, the application of the Choquet integral, as well as the application of all non-additive methods mentioned above, involves some problems related to:

1) the determination of the capacity representing the interaction between criteria,

2) the construction of a common scale permitting comparisons between evaluations on different criteria.

To handle point 1), we propose to use the Non Additive Robust Ordinal Regression (NAROR) [4] that considers the whole set of capacities compatible with the preference information provided by the DM while, to handle point 2) we propose to use the Analytic Hierarchy Process (AHP, [56, 57]). Let us spend some words to give the intuition behind our proposal. We shall give more details on how to deal with the two mentioned problems and on the reasons of combining them together in the following sections of the paper.

In any MCDA problem, a decision model has to be built to produce a recommendation and its preference parameters (weights, thresholds, value functions and so on) have to be determined. This is usually done in cooperation with the DM, who can give directly the preference parameters or, instead, can supply some preference information, for example in terms of preference pairwise comparison of some alternatives, from which preference parameters can be induced. In the case of the Choquet integral model, the preference parameters to be fixed are the weights that the capacity assigns to 
each one of the $2^{n}$ subsets of a family of $n$ criteria (for example $2^{10}=1024$ weights for a family of 10 criteria). Due to this so huge number of parameters, very often the values assigned by the capacity to the subset of criteria are not asked directly to the DM and, consequently, several methodologies have been proposed to determine a capacity compatible with the preference information provided by the DM. For example, in [34] four different approaches are presented to deal with this problem and there is no general suggestion about which one to adopt. In this conditions, it seems very wise to take into account not one among the many capacities compatible with the DM's preference information, but, instead, the whole set of capacities compatible with the available preference information. This is the aim of the NAROR that is based on the concepts of necessary and possible preferences, holding between two alternatives $a$ and $b$ if $a$ is at least as good as $b$ for all or for at least one capacity compatible with the preferences provided by the DM.

In addition to the determination of the capacity, another important issue regarding the application of the Choquet integral is the building of a common scale on which evaluations of alternatives on considered criteria can be compared. Observe that also in this respect it is not reasonable to ask directly to the DM the values that have to be assigned to the evaluations of criteria at hand. Indeed, the DM is not able to take into consideration at the same time all the evaluations that criteria give to all alternatives and to put everything in a single scale. For this reason, we remember the famous article of Miller "The Magical Number Seven, Plus or Minus Two: Some Limits on Our Capacity for Processing Information" [47] in which it is argued that the average human brain can handle a number of objects equal to $7 \pm 2$. More precisely, Miller showed that this is due to the limits of onedimensional absolute judgment and to the limits of short-term memory. This suggests to consider pairwise comparisons among objects in consequence of their manageability for the human mind, and to use some proper methodology permitting to represent pairwise comparisons by numerical evaluations of objects at hand. Some experiments showed that the use of pairwise comparisons and, even better, verbal pairwise comparisons, is much more accurate than direct estimation [48]. Consequently, to construct the common scale we propose to adopt AHP which is the most well known methodology used to build priority vectors on a homogeneous scale on the basis of pairwise comparisons of alternatives with respect to considered criteria. Some limits related to the capacity of identifying possible sources of inconsistency arise in case the number of objects to be compared is larger than seven [59]. Consequently, taking also into account the cognitive burden required to compare pairwise all the alternatives with respect to all considered criteria, we propose to focus the attention of the DM on a selected number of meaningful reference levels on each criterion. The DM is asked to supply pairwise comparisons of reference evaluations, so that they can be put on a single scale through AHP. All other evaluations are put on the common scale through linear interpolation. With this approach we reduce the effort asked to the DM for supplying the preference information and we increase the reliability of the results by taking into account pairwise comparisons given in a much more careful way (because the number of required comparisons is much smaller and the reference levels to be compared are meaningful for the DM that is supposed to participate to their selection).

Recently, some contributions proposed to conjugate AHP and the Choquet integral [8, 9, 66]. Our approach can be compared with these three works as follows:

1. [9] and [66] use AHP within a procedure to determine the capacity. More precisely, [9] uses AHP to determine a priority vector of the criteria, taking into account a capacity representing inconsistencies in the pairwise comparison matrix. Instead, [66] uses AHP to evaluate the importance of each criterion in terms of the Shapley index and, after asking the DM to supply the interactions degree of each couples of criteria, uses a nonlinear programming to get the capacity. With respect to these contributions, the method we are proposing presents a radical difference because we use NAROR, and not AHP, to define the whole set of capacities compatible with the DM's preference information containing pairwise comparisons of some real alternatives for 
which the DM is confident in expressing her own convictions;

2. [8] uses a pairwise comparison method to put on a common scale the evaluations of alternatives with respect to considered criteria (in fact it uses MACBETH, but AHP can be used as well) and determines the capacity on the basis of the comparisons supplied by the DM of some fictitious situations where the criteria have evaluations either totally satisfactory or totally unsatisfactory. This approach is closer to our approach but:

- it uses a pairwise comparison method (which can be AHP as well as MACBETH) to construct evaluations of alternatives on considered criteria on the basis of pairwise comparisons of all the alternatives which can be very numerous and requiring, therefore, a strong cognitive effort to the DM; instead, in our method we consider comparisons of few reference levels for each criterion and from the evaluations of these reference levels, by linear interpolation, we assign evaluations being on a common scale to the alternatives at hand with respect to considered criteria;

- it determines the capacity considering very specific fictitious alternatives while we can consider any alternative, so that the DM can choose those ones that she knows best and for which she feels more comfortable in expressing her preferences. Moreover, it determines only one capacity while we consider the whole set of capacities compatible with the preference information provided by the DM.

The paper is organized as follows. In the next section, we state the problem and we give the basic intuition of our proposal. Section 3 recalls the basic concepts of the Choquet integral and NAROR and it gives fundamental notions of AHP. It also introduces our methodology to build the common scale required by the Choquet integral by means of the AHP. Section 4 illustrates how our approach can be applied in a multiple criteria decision problem. Section 5 collects conclusions and further directions of research.

\section{Problem statement and intuition}

To explain the idea behind the Choquet integral, inspired by [31], we consider and discuss in detail a problem of evaluation of students. The Dean of a high school has to evaluate students with respect to three subjects: Mathematics (Math), Physics (Phys) and Literature (Lit). He starts using a simple weighted sum whose weights represent the importance of the different subjects. Supposing that Mathematics and Physics are more important than Literature, the weights could be 3, 3 and 2, i.e., after normalization, $w_{\text {Math }}=w_{\text {Phys }}=\frac{3}{8}=0.375, w_{\text {Lit }}=\frac{2}{8}=0.25$, respectively. Let us consider students $A, B, C$ and $D$ having their scores given on a $0-20$ scale as shown in Table 1 . The next to the last column of Table 1 gives the weighted sum of the four students at hand considering the weights of criteria previously defined.

Table 1: Evaluations of the students on the three considered criteria

\begin{tabular}{|l|c|c|c|c|c|}
\hline Student/Subjects & Mathematics (Math) & Physics (Phys) & Literature (Lit) & Weighted Sum & Choquet integral \\
\hline A & 18 & 16 & 14 & 16.25 & 15.9 \\
B & 18 & 14 & 16 & 16 & 16.7 \\
C & 14 & 16 & 14 & 14.75 & 14.9 \\
D & 14 & 14 & 16 & 14.5 & 14.6 \\
\hline
\end{tabular}

Applying the weighted sum, student $A$ is preferred to student $B$ and student $C$ to student $D$. The Dean agrees with the preference of $C$ over $D$, but he thinks that the preference of $A$ over $B$ can be questionable. Indeed student $A$ has relatively good scores both in Mathematics and Physics, while he has a weakness in Literature. Mathematics and Physics are both important subjects, but students 
with a good score in Mathematics have generally also a good score in Physics and, consequently, there is an overvaluation of these students, in this case of student $A$. On the other hand, students good in Mathematics (or Physics) are generally not good in Literature; therefore, the Dean wants to give a bonus to student $B$ because he has good scores in Mathematics and Literature, even if he has a weakness in Physics. The Dean wonders if it is possible to represent his preferences by changing the weights assigned to the considered subjects. Unfortunately, this is not possible because the weighted sum cannot represent the preferences of the Dean with respect to the four students. Indeed, on one hand, the preference of $B$ over $A$ should imply that

$$
18 w_{\text {Math }}+16 w_{\text {Phys }}+14 w_{\text {Lit }}<18 w_{\text {Math }}+14 w_{\text {Phys }}+16 w_{\text {Lit }},
$$

while, on the other hand, the preference of $C$ over $D$ should imply that

$$
14 w_{\text {Math }}+16 w_{\text {Phys }}+14 w_{\text {Lit }}>14 w_{\text {Math }}+14 w_{\text {Phys }}+16 w_{\text {Lit }} .
$$

By (1) we get

$$
16 w_{\text {Phys }}+14 w_{\text {Lit }}<14 w_{\text {Phys }}+16 w_{\text {Lit }},
$$

while, by (2) we get

$$
16 w_{\text {Phys }}+14 w_{\text {Lit }}>14 w_{\text {Phys }}+16 w_{\text {Lit }}
$$

and, clearly, (3) and (4) are incompatible. The Dean considers also the possibility to evaluate students using an additive value function such as

$$
u_{\text {Math }}\left(\text { score }_{\text {Math }}\right)+u_{\text {Phys }}\left(\text { score }_{\text {Phys }}\right)+u_{\text {Lit }}\left(\text { score }_{\text {Lit }}\right),
$$

with $u_{\text {Math }}, u_{\text {Phys }}$ and $u_{\text {Lit }}$ being non-decreasing in their arguments. Also additive value functions cannot represent the preferences of the Dean with respect to the four students $A, B, C$ and $D$. In fact, the preference of $B$ over $A$ should imply that

$$
u_{\text {Math }}(18)+u_{\text {Phys }}(16)+u_{\text {Lit }}(14)<u_{\text {Math }}(18)+u_{\text {Phys }}(14)+u_{\text {Lit }}(16) \text {, }
$$

while, the preference of $C$ over $D$ should imply that

$$
u_{\text {Math }}(14)+u_{\text {Phys }}(16)+u_{\text {Lit }}(14)>u_{\text {Math }}(14)+u_{\text {Phys }}(14)+u_{\text {Lit }}(16) .
$$

By (6) we get

$$
u_{\text {Phys }}(16)+u_{\text {Lit }}(14)<u_{\text {Phys }}(14)+u_{\text {Lit }}(16) \text {, }
$$

while, by (7) we get

$$
u_{\text {Phys }}(16)+u_{\text {Lit }}(14)>u_{\text {Phys }}(14)+u_{\text {Lit }}(16),
$$

and, again, (8) and (9) are incompatible. In fact, the preferences of the Dean do not respect preference independence [45] that would require that for alternatives $a, b, c, d \in A$ if

- $g_{i}(a)=g_{i}(b)$ and $g_{i}(c)=g_{i}(d)$ for $g_{i} \in G^{\prime} \subset G$,

- $g_{i}(a)=g_{i}(c)$ and $g_{i}(b)=g_{i}(d)$ for $g_{i} \in G \backslash G^{\prime}$,

- $a$ is preferred to $b$,

then also $c$ should be preferred to $d$. In simple words, preference independence would require that if two alternatives $a$ and $b$ have the same evaluation on a subset of criteria $G^{\prime} \subset G$, then the preference of the DM should depend only on the evaluations with respect to remaining criteria, that is criteria in $G \backslash G^{\prime}$, regardless the evaluation on criteria from $G^{\prime}$. This would imply that if any 
two other alternatives $c$ and $d$ have the same evaluations on criteria from $G^{\prime}$ (even if different from the evaluations got by $a$ and $b$ on the same criteria) and on criteria from $G \backslash G^{\prime} c$ has the same evaluations of $a$ and $d$ has the same evaluations of $b$, then $c$ must be preferred to $d$. When this is not the case, the level of the evaluations on criteria $G^{\prime}$ is relevant for the preference of an alternative over the other, even if these evaluations are the same for the two compared alternatives. In these cases, preference independence is violated. Observe that this situation applies to students $A, B, C$ and $D$. Indeed, $A$ and $B$ have the same score in Mathematics and therefore one could imagine that the Dean's preference of $B$ over $A$ should depend only on the scores on Physics and Literature. But if this would be true, then the Dean should prefer also $D$ to $C$, because in Physics and Literature also $C$ and $D$ have the same scores of $A$ and $B$, respectively, while they have the same score in Mathematics. However, the common score in Mathematics of $C$ and $D(14)$ is different from the common score in the same subject of $A$ and $B(18)$. Consequently $C$ and $D$ have a weakness in Mathematics, while $A$ and $B$ have a quite good score in the same subject. Thus, it is absolutely reasonable that the perspective of the Dean changes in evaluating $C$ and $D$ on one hand and $A$ and $B$ on the other hand. Comparing students $C$ and $D$, since their scores in Mathematics is not so good, taking into account the technical orientation of the school, the Dean prefers $C$ over $D$ for her relatively good score in Physics. Instead, comparing students $A$ and $B$, since their score in Mathematics is good, taking into account a favor for well equilibrated students in Science (Mathematics and Physics) and Humanities (Literature), the Dean prefers $B$ over $A$ for his good score in Literature. In conclusion, the preferences of the Dean violate preference independence and there are sound reasons for this. Reflecting on the problem, the Dean arrives at the conclusion that one should consider a weight not only for each subject, but also for each subset of subjects in order to represent:

- the redundancy between Mathematics and Physics; in this case the weight given to Mathematics and Physics together should be smaller than the sum of the weights given to Mathematics and Physics considered alone;

- the synergy between Mathematics and Literature or Physics and Literature; in this case the weight given to Mathematics and Literature together should be greater than the sum of the weights given to Mathematics and Literature considered alone; analogous behavior should have the weights of Physics and Literature.

Therefore, denoting by $\mu(S)$ the weight of the subset of subjects $S$, we could consider the following weights:

$$
\begin{gathered}
\mu(\{\text { Math }\})=\mu(\{\text { Phys }\})=0.45, \mu(\{\text { Lit }\})=0.3, \\
\mu(\{\text { Math, Phys }\})=0.5, \quad \mu(\{\text { Math }, \text { Lit }\})=\mu(\{\text { Phys, Lit }\})=0.9
\end{gathered}
$$

and, of course,

$$
\mu(\{\text { Math }, \text { Phys }, \text { Lit }\})=1 .
$$

It can be observed that:

- according to the redundancy between Mathematics and Physics,

$$
\mu(\{\text { Math }\})+\mu(\{\text { Phys }\})>\mu(\{\text { Math, Phys }\}),
$$

- according to the synergy between Mathematics and Literature,

$$
\mu(\{\text { Math }\})+\mu(\{\text { Lit }\})<\mu(\{\text { Math }, \text { Lit }\}),
$$


- according to the synergy between Physics and Literature,

$$
\mu(\{\text { Phys }\})+\mu(\{\text { Lit }\})<\mu(\{\text { Phys, Lit }\}) .
$$

Now the problem is: how to extend the weighted sum in case of interacting criteria? In other words: how to redefine the weighted sum in order to take into account not only weights for each subject (criterion), but also for each subset of subjects? Let us introduce the Choquet integral [14] explaining why it can be considered as an extension of the weighted sum in case of interacting criteria. Indeed, in this case we have to consider not only a weight for each one of the considered criteria, but also a weight for each subset of considered criteria. Let us consider the case of a set of $n$ non-negative values $x_{1}, \ldots, x_{n}$ for which a weighted sum has to be computed on the basis of the weights $w_{1}, \ldots, w_{n}$, $w_{i} \geq 0$ and $w_{1}+\ldots+w_{n}=1$, where $w_{i}$ represents the importance of the value (criterion) $x_{i}$. The weighted sum is given by

$$
W S\left(x_{1}, \ldots, x_{n} ; w_{1}, \ldots, w_{n}\right)=w_{1} x_{1}+\ldots+w_{n} x_{n} .
$$

Observe that we can re-write the weighted sum as follows

$$
W S\left(x_{1}, \ldots, x_{n} ; w_{1}, \ldots, w_{n}\right)=\sum_{i=1}^{n}\left[\left(x_{(i)}-x_{(i-1)}\right) \sum_{j=i}^{n} w_{(j)}\right]
$$

where $(\cdot)$ is a permutation of the indices $1, \ldots, n$ such that $x_{(1)} \leq \ldots \leq x_{(n)}$ and $x_{(0)}=0$. For example, considering student $A$, according to (10) we have

$$
W S(18,16,14 ; 0.375,0.375,0.25)=0.375 \cdot 18+0.375 \cdot 16+0.25 \cdot 14=16.25
$$

while, according to (11) we have

$$
\begin{gathered}
W S(18,16,14 ; 0.375,0.375,0.25)= \\
=(14-0) \cdot(0.375+0.375+0.25)+(16-14) \cdot(0.375+0.375)+(18-16) \cdot 0.375=16.25 .
\end{gathered}
$$

In case of absence of interaction between criteria, the importance of a subset of criteria is

$$
\mu(S)=\sum_{i \in S} w_{i}
$$

so that

$$
\sum_{j=i}^{n} w_{(j)}=\mu(\{(i), \ldots,(n)\})
$$

and (11) can be written as follows:

$$
W S\left(x_{1}, \ldots, x_{n} ; w_{1}, \ldots, w_{n}\right)=\sum_{i=1}^{n}\left[\left(x_{(i)}-x_{(i-1)}\right) \mu(\{(i), \ldots,(n)\})\right] .
$$

Now the Choquet integral, denoted by $C_{\mu}\left(x_{1}, \ldots, x_{n}\right)$, is formulated exactly as (12), i.e.

$$
C_{\mu}\left(x_{1}, \ldots, x_{n}\right)=\sum_{i=1}^{n}\left[\left(x_{(i)}-x_{(i-1)}\right) \mu(\{(i), \ldots,(n)\})\right]
$$


and this formulation holds also in case of interacting criteria.

Therefore, according to (13), considering the capacity defined above, the Choquet integral of the scores of student $A$ is

$$
\begin{aligned}
C_{\mu}(18,16,14)= & (14-0) \cdot \mu(\{M, P, L\})+(16-14) \cdot \mu(\{M, P\})+(18-16) \cdot \mu(\{M\})= \\
& =(14-0) \cdot 1+(16-14) \cdot 0.5+(18-16) \cdot 0.45=15.9 .
\end{aligned}
$$

The Choquet integral of the scores of students $B, C$ and $D$ can be computed analogously obtaining the results shown in the last column of Table 1. One can see that, in this case, student $B$ is evaluated better than student $A$ and student $C$ is evaluated better than student $D$.

We have shown that the Choquet integral is able to take into account interactions between criteria. However, the application of the Choquet integral presents two relevant problems that did not appear clearly in the above didactic example:

a) Differently from the usual weighted sum, where we have to assign only one weight to each criterion, the Choquet integral requires to assign a weight to each subset of criteria. This problem becomes very relevant when the number of criteria is high. Indeed, for $n$ criteria we have to assign $n$ values in case of the weighted sum (one for each criterion), while we have to assign $2^{n}$ weights (one for each subset of criteria) in case of the Choquet integral (in fact $2^{n}-2$ weights because the weight assigned to the empty set is null and the weight assigned to the whole set of criteria is equal to one). For example, if we have 3 criteria, we have to assign $2^{3}=8$ weights which become $2^{4}=16$ if we have 4 criteria and so on. It is clear that asking to the DM to provide a large number of weights is not reasonable. It is also worthwhile to observe that the interpretation of these weights is not trivial for the DM because of the interaction of several criteria at once.

b) As it can be seen from (11), the Choquet integral requires that the evaluations with respect to all considered criteria are on the same scale. In fact, for each alternative, the evaluations on the considered criteria need to be ordered from the smallest to the greatest. In the example of Table 1, if we consider student $A$, it is immediate to conclude that a score of 18 in Mathematics is greater than a score of 16 in Physics which, in turn, is greater than a score of 10 in Literature. Even more, as shown by (13), to compute the Choquet integral we have also to consider the difference between evaluations of the same alternative on different criteria. Therefore, for example, taking into consideration student $A$, we must be able to say that the difference in the score between Mathematics and Physics is $18-16=2$, and that it is meaningful to state that it is one half of the difference between Mathematics and Literature which is $18-14=4$. However, let suppose we have to rank a set of cars to decide which one to buy and suppose we want to use the Choquet integral to evaluate, for example, Audi A3 (3 doors) having the following characteristics:

Price: $€ 22,140$

Acceleration: 10.3 second to arrive from 0 to $100 \mathrm{~km} / \mathrm{h}$

Maximum speed: $193 \mathrm{~km} / \mathrm{h}$

Fuel consumption: $4.9 \mathrm{l} / \mathrm{km}$.

How to order these evaluations for computing the Choquet integral? In other words, is a price of $€ 22,140$ more valuable than a maximum speed of $193 \mathrm{~km} / \mathrm{h}$ ? And, even much more problematic: how can we give a value to this difference?

Some answers have been given in the literature to the two above points. Regarding point a), the most convincing answer seems the proposal of inducing the weights from some indirect preference 
information provided by the DM in terms of pairwise comparisons of some reference alternatives and in terms of relative importance and interaction between criteria [46]. Recently, in the same direction, NAROR [4] has been proposed. It permits to consider the whole set of compatible weights, i.e. the whole set of weights satisfying the preference information provided by the DM, by the use of a necessary and a possible preference relation as in any Robust Ordinal Regression method (ROR; $[16,17,37])$. The necessary and possible preference relations hold between alternatives $a$ and $b$ if $a$ is not worse than $b$ for all, or for at least one, of the sets of weights compatible with the DMs preferences, respectively (for a discussion on the axiomatic basis of necessary and possible preference relations see [27]).

With respect to point b), very often a normalization of evaluations on each criterion is done considering an "unacceptable" minimal value and an "ideal" maximal value for each criterion and considering a linear interpolation between these two extremes. A more sophisticated methodology permitting to build one scale and one capacity for the Choquet integral on the basis of the preference information provided by the DM has been proposed in [2] and it has been further developed in [1]. However, these two approaches are heuristics and not exact algorithms.

In this paper we propose to deal with problems a) and b) in a systematic way as follows:

- First we construct a common scale for all criteria using the AHP. The advantage of using AHP in this context is given by the possibility of building the scale using the preferences provided by the DM. Moreover, for the considered criteria, the evaluations on the scale obtained by AHP are comparable between them and therefore we can apply the Choquet integral; it is worthwhile to observe that our use of AHP is parsimonious with respect to the information asked to the DM, in the sense that with respect to each considered criterion, we shall not ask the DM to compare pairwise all the alternatives, as it is commonly done, but we shall ask the DM to compare some reference levels on the considered criteria. The other non reference evaluations are obtained by interpolating the values assigned by AHP to the reference levels. We believe that this is another important contribution of our work which goes beyond the mere use of AHP in a MCDA procedure based on the Choquet integral preference model. In fact, it can be used in any decision problem where AHP has to be applied to a large set of alternatives. In this way, the DM avoids to answer to a long and tiring list of questions related to all pairwise comparisons of the alternatives at hand.

- Using the evaluations expressed in the scale obtained by means of the AHP method, we proceed with the application of the NAROR. In this way, we consider all the set of weights which are compatible with the preference information provided by the DM avoiding to consider only one set of weights chosen in an arbitrary way in the whole family of compatible sets. Since the necessary and possible preference relations obtained by NAROR can present some difficulty to be handled by the DM, we present her also a complete ranking of the considered alternatives obtained by computing the so called most representative value function [3,22]. This is a value function corresponding to the Choquet integral with respect to the capacity giving the "best" representation not only of all the preferences supplied by the DM, but also of the necessary and the possible preference relations.

We believe that the proposed combination of AHP and NAROR represents the most convenient way to deal with the two discussed problems of determining in a reasonable and meaningful way a set of weights and a common scale for the considered criteria when the Choquet integral is used to represent interaction between criteria. 


\section{Methodology}

\subsection{Introduction}

This section presents the methods used to solve the problem described in Section 2. First, we formalize the Choquet integral preference model for MCDA problems (Section 3.2). As the capacities to be used in the Choquet integral are difficult to elicit, we use the NAROR (Section 3.3), to indirectly infer all capacities compatible with the information provided by the DM. In Section 3.4.1 basic principles of AHP are then recalled, describing after how to use it for building the common scale, which is needed to apply the Choquet integral. In Section 3.4.2 we show how the number of pairwise comparisons asked by the AHP can be decreased by taking into account only reference points and then interpolating the results.

\subsection{The Choquet integral preference model}

A set function $\mu: 2^{G} \rightarrow[0,1]$ is called a capacity (fuzzy measure) on $2^{G}$ (being the power set of $G$, i.e. the set of all subsets of $G$ ) if the following properties hold:

1a) $\mu(\emptyset)=0$ and $\mu(G)=1$ (boundary conditions),

2a) $\forall S \subseteq T \subseteq G, \mu(S) \leq \mu(T)$ (monotonicity condition).

For any $T \subseteq G, \mu(T)$ represents the total weight of criteria from $T$, which is not supposed to be additive, i.e. it is not necessarily true that for any $S, T \subseteq G$ such that $S \cap T=\emptyset$, one has $\mu(S \cup T)=\mu(S)+\mu(T)$.

In this case, we have to define $2^{|G|}-2$ non additive weights $\mu(S), \emptyset \subset S \subset G$, since the values $\mu(\emptyset)=0$ and $\mu(G)=1$ are already known.

Given $a \in A$ and a capacity $\mu$ on $2^{G}$, the Choquet integral [14], as above explained, gives the analogous of the weighted sum in case of additive weights, and it is defined as follows:

$$
C_{\mu}(a)=\sum_{i=1}^{n}\left[\left(g_{(i)}(a)-g_{(i-1)}(a)\right) \mu(\{(i), \ldots,(n)\})\right]
$$

where $(\cdot)$ reorders the criteria so that $g_{(1)}(a) \leq \ldots \leq g_{(n)}(a)$ and $g_{(0)}(a)=0$.

It is useful to consider also the Möbius representation of a capacity $\mu$ being the function $m: 2^{G} \rightarrow \mathbb{R}$ $[53,61]$ such that, for all $S \subseteq G$

$$
\mu(S)=\sum_{T \subseteq S} m(T)
$$

The Möbius representation $m(S)$ can be obtained from $\mu(S)$ as follows:

$$
m(S)=\sum_{T \subseteq S}(-1)^{|S-T|} \mu(T) .
$$

For the Möbius representation [13], properties 1a) and 2a) become

1b) $m(\emptyset)=0, \quad \sum_{T \subseteq G} m(T)=1$,

2b) $\forall i \in G$ and $\forall R \subseteq G \backslash\{i\}, m(\{i\})+\sum_{T \subseteq R} m(T \cup\{i\}) \geq 0$. 
The Möbius representation is important in applications, because it permits to express the Choquet integral in a linear form (but in a space different from that one of values given by criteria from $G$ ), formulating the Choquet integral as a weighted sum of minimum values given to the considered alternative $a \in A$ by all subsets of criteria $T$ from $G$ [29],

$$
C_{\mu}(a)=\sum_{T \subseteq G} m(T) \min _{i \in T} g_{i}(a)
$$

However, even if expressed in linear form with the above formula, the use of the Choquet integral preference model remains difficult because we have to determine the $2^{|G|}$ values $m(T), T \subseteq G$. In order to reduce the number of parameters to be determined and to get a simpler formulation for the Choquet integral, the concept of $k$-additive capacity, $k=1, \ldots, n$, has been introduced [32]. Formally a capacity is $k$-additive if $m(T)=0$ for $T \subseteq G$ such that $|T|>k$. Intuitively a capacity is $k$-additive if it considers interactions between no more than $k$ criteria. In MCDA, 2-additive capacities are often considered because it is reasonable to expect that the DM could supply preference information on positive and negative interactions between couples of criteria, while it seems that interactions between three, four and more criteria are more difficult, or even sometimes impossible, to evaluate. It is to observe that a 2-additive capacity $\mu$ in terms of Möbius representation has the following formulation

$$
\mu(S)=\sum_{i \in S} m(\{i\})+\sum_{\{i, j\} \subseteq S} m(\{i, j\}), \forall S \subseteq G .
$$

Thus, from the computational point of view, 2-additive capacities require to induce the value of only $n+\left(\begin{array}{l}n \\ 2\end{array}\right)$ parameters, being a value $m(\{i\})$ for every criterion $i$ and a value $m(\{i, j\})$ for every couple of criteria $\{i, j\}$. For 2 -additive capacities, properties $1 \mathrm{~b}$ ) and $2 \mathrm{~b}$ ) have to be reformulated as follows:

1c) $m(\emptyset)=0, \sum_{i \in G} m(\{i\})+\sum_{\{i, j\} \subseteq G} m(\{i, j\})=1$

2c) $\left\{\begin{array}{l}m(\{i\}) \geq 0, \forall i \in G, \\ m(\{i\})+\sum_{j \in T} m(\{i, j\}) \geq 0, \forall i \in G \text { and } \forall T \subseteq G \backslash\{i\}, T \neq \emptyset .\end{array}\right.$

When the Choquet integral is adopted in MCDA, the importance of a criterion $i \in G$ is not evaluated considering only the value assigned by the capacity to the criterion $i$ alone, i.e. $\mu(\{i\})$, but also taking into consideration all its interactions, i.e., in case of 2 -additive capacities, considering $\mu(\{i, j\})$ for all $j \in G \backslash\{i\}$. So doing, the importance of criterion $i \in G$ is expressed by the Shapley value [62] that, in case of a 2-additive capacity, has the following formulation:

$$
\varphi(\{i\})=m(\{i\})+\sum_{j \in G \backslash\{i\}} \frac{m(\{i, j\})}{2} .
$$

As pointed out above, with respect to a criterion $i \in G$, in general the Shapley index $\varphi(\{i\})$ is different from the weight $\mu(\{i\})$ assigned to the criterion $i$ by the capacity $\mu$. Our methodology takes into account this fact by modeling DM's preference information related to comparison between the importance of criteria in terms of Shapley index $\varphi(\{i\})$. In this way, we acknowledge that the importance of criterion $i$ does not depend on itself only but also on its interactions with the other criteria at hand.

Among the preference information that the DM can supply there is also the sign and the magnitude of the interaction $\varphi(\{i, j\})$ of couples of criteria $\{i, j\} \subseteq G$ [50]. For a 2-additive capacity $\mu$ the interaction is given by the Möbius representation of the couple $\{i, j\}$, i.e. 


$$
\varphi(\{i, j\})=m(\{i, j\}) .
$$

\subsection{Robust Ordinal Regression and NAROR}

\subsubsection{Intuition of NAROR}

Consider the example proposed in Section 2. Suppose now that the Dean wants to evaluate three new students $E, F$ and $H$, whose scores are shown in Table 2.

Table 2: Evaluations of three new students on the three considered criteria

\begin{tabular}{|l|c|c|c|c|c|}
\hline Student/Subjects & Mathematics (M) & Physics (P) & Literature (L) & Choquet integral with respect to capacity $\mu_{1}$ & Choquet integral with respect to capacity $\mu_{2}$ \\
\hline $\mathrm{E}$ & 19 & 14 & 15 & 16.48 & 16.3125 \\
$\mathrm{~F}$ & 18 & 18 & 14 & 16 & 16.5 \\
$\mathrm{H}$ & 18 & 14 & 18 & 16.4 & 17.25 \\
\hline
\end{tabular}

The Dean wants to apply the Choquet integral but he wants to be sure about his evaluations, and, consequently, he wants to consider all the capacities $\mu$ that are coherent with his preferences. Therefore he takes into account the following constraints for the values taken by $\mu$ :

- $\mu(\{$ Math, Lit $\})>\mu(\{$ Math, Phys $\})$;

Indeed, by considering the preference of B over A translated by the inequality

$$
C_{\mu}(18,14,16)>C_{\mu}(18,16,14)
$$

and applying (13), we have that

$$
\begin{gathered}
14 \mu(\{\text { Math, Phys, Lit }\})+(16-14) \mu(\{\text { Math }, \text { Lit }\})+(18-16) \mu(\{\text { Math }\}) \\
> \\
14 \mu(\{\text { Math, Phys, Lit }\})+(16-14) \mu(\{\text { Math, Phys }\})+(18-16) \mu(\{\text { Math }\})
\end{gathered}
$$

and, consequently,

$$
\mu(\{\text { Math }, \text { Lit }\})>\mu(\{\text { Math, Phys }\}) .
$$

- $\mu(\{$ Phys $\})>\mu(\{$ Lit $\})$;

Indeed, by considering the preference of $\mathrm{C}$ over $\mathrm{D}$ translated by the inequality,

$$
C_{\mu}(14,16,14)>C_{\mu}(14,14,16)
$$

and applying (13) we have that

$$
\begin{gathered}
14 \mu(\{\text { Math, Phys, Lit }\})+(16-14) \mu(\{\text { Phys }\}) \\
> \\
14 \mu(\{\text { Math, Phys, Lit }\})+(16-14) \mu(\{\text { Lit }\})
\end{gathered}
$$

and, consequently,

$$
\mu(\{\text { Phys }\})>\mu(\{\text { Lit }\}) .
$$

$$
\mu(\{\text { Math }\})=\mu(\{\text { Phys }\}) \text { and } \mu(\{\text { Math }, \text { Lit }\})=\mu(\{\text { Phys, Lit }\})
$$

because Mathematics and Physics are considered equally important by the Dean. 
Considering all the capacities $\mu$ satisfying constraints (14)-(16), the Dean arrives at the following conclusions:

- student $H$ is preferred to student $F$ for every capacity $\mu$ compatible with his preferences; indeed

$$
C_{\mu}(18,14,18)>C_{\mu}(18,18,14)
$$

that, by (13), becomes

$$
\begin{aligned}
14 \mu(\{\text { Math, Phys, Lit }\}) & +(18-14) \mu(\{\text { Math }, \text { Lit }\}) \\
& > \\
14 \mu(\{\text { Math, Phys, Lit }\})+ & (18-14) \mu(\{\text { Math, Phys }\})
\end{aligned}
$$

which is always true by eq. (14);

- among the capacities $\mu$ compatible with his preferences, there are some for which student $E$ is preferred to student $F$ and there are others for which student $F$ is preferred to student $E$; for example, student $E$ is preferred to student $F$ for the capacity $\mu_{1}$ such that

$$
\begin{gathered}
\mu_{1}(\{\text { Math }, \text { Lit }\})=\mu_{1}(\{\text { Phys }, \text { Lit }\})=0.6, \mu_{1}(\{\text { Math, Phys }\})=0.5, \\
\mu_{1}(\{\text { Math }\})=\mu_{1}(\{\text { Phys }\})=0.47, \mu_{1}(\{\text { Lit }\})=0.1, \mu_{1}(\{\text { Math, Phys, Lit }\})=1,
\end{gathered}
$$

while, student $F$ is preferred to student $E$ for the capacity $\mu_{2}$ such that

$$
\begin{gathered}
\mu_{2}(\{\text { Math }, \text { Lit }\})=\mu_{2}(\{\text { Phys }, \text { Lit }\})=0.8125, \mu_{2}(\{\text { Math, Phys }\})=0.625, \\
\mu_{2}(\{\text { Math }\})=\mu_{2}(\{\text { Phys }\})=0.375, \mu_{2}(\{\text { Lit }\})=0.1875, \mu_{2}(\{\text { Math, Phys, Lit }\})=1 ;
\end{gathered}
$$

the evaluations given to students $E, F$ and $H$ by the Choquet integral with respect to capacities $\mu_{1}$ and $\mu_{2}$ are shown in the last two columns of Table 2 ;

- among the capacities $\mu$ compatible with his preferences, there are some for which student $E$ is preferred to student $H$ and there are others for which student $H$ is preferred to student $E$; for example, student $E$ is preferred to student $H$ for the capacity $\mu_{1}$ while, student $H$ is preferred to student $E$ for the capacity $\mu_{2}$.

In conclusion, the Dean is convinced that there is no doubt about the preference of student $H$ over student $F$. In this case, we speak of necessary preference. However, there are some doubts about the preference between student $E$ and student $F$, and between student $E$ and student $H$. In this case we speak of possible preferences. The following subsection 3.3 recalls basic concepts of NAROR [4] that permits to define systematically necessary and possible preferences when using the Choquet integral.

\subsubsection{The formal model of $N A R O R$}

As observed in the example of the previous Section, in general, there is more than one capacity that permits to represent the preference information provided by the DM through the Choquet integral. Since choosing only one of these compatible capacities is always arbitrary to some extent, following the principles of ROR [16, 17, 37], we take into account all the capacities compatible with the preference information provided by the DM through NAROR [4] that we shall recall in the following. To get the values $\mu(T)$ that the capacity $\mu$ assigns to all the subsets $T$ of $G$, one can use a direct or an indirect technique. The direct technique asks the values $\mu(T)$ or the corresponding Möbius representation directly to the DM while the indirect technique infers the values $\mu(T)$ from some 
preference information provided by the DM $([2,46]$; for an extensive review on the topic see also [33] and $[35])$.

When using an indirect technique, the DM can supply the following information with respect to a subset of alternatives $A^{R} \subseteq A$ :

- a partial preorder $\succsim$ on $A^{R}$ whose meaning is: for $a^{*}, b^{*} \in A^{R}$

$$
a^{*} \succsim b^{*} \Leftrightarrow \text { " } a^{*} \text { is at least as good as } b^{*} \text {; }
$$

- a partial preorder $\succsim^{*}$ on $A^{R} \times A^{R}$, whose meaning is: for $a^{*}, b^{*}, c^{*}, d^{*} \in A^{R}$,

$$
\left(a^{*}, b^{*}\right) \succsim^{*}\left(c^{*}, d^{*}\right) \Leftrightarrow \text { " } a^{*} \text { is preferred to } b^{*} \text { at least as much as } c^{*} \text { is preferred to } d^{*} \text {; }
$$

- a partial preorder $\succsim_{1}$ on $G$, whose meaning is: for $g_{i}, g_{j} \in G$

$$
g_{i} \succsim_{1} g_{j} \Leftrightarrow \text { "criterion } g_{i} \text { is at least as important as criterion } g_{j} \text { "; }
$$

- a partial preorder $\succsim_{1}^{*}$ on $G \times G$, whose meaning is: for $g_{i}, g_{j}, g_{k}, g_{l} \in G$, $\left(g_{i}, g_{j}\right) \succsim_{1}^{*}\left(g_{k}, g_{l}\right) \Leftrightarrow$ "the difference of importance between criteria $g_{i}$ and $g_{j}$ is no lower than the difference of importance between criteria $g_{k}$ and $g_{l} "$;

- the sign of the interaction between criteria $g_{i}$ and $g_{j}$, with $g_{i}, g_{j} \in G$ :

(a) $g_{i}$ and $g_{j}$ are positively interacting,

(b) $g_{i}$ and $g_{j}$ are negatively interacting.

In the following, as usual, $\sim$ (indifference) and $\succ$ (preference) so as $\sim^{*}$ and $\succ^{*}$ denote the symmetric and the asymmetric part of $\succsim$ and $\succsim^{*}$, respectively, that is,

- $a^{*} \sim b^{*}$ is equivalent to $a^{*} \succsim b^{*}$ and $b^{*} \succsim a^{*}$, while

- $a^{*} \succ b^{*}$ is equivalent to $a^{*} \succsim b^{*}$ and $\operatorname{not}\left(b^{*} \succsim a^{*}\right)$,

as well as

- $\left(a^{*}, b^{*}\right) \sim^{*}\left(c^{*}, d^{*}\right)$ is equivalent to $\left(a^{*}, b^{*}\right) \succsim^{*}\left(c^{*}, d^{*}\right)$ and $\left(c^{*}, d^{*}\right) \succsim^{*}\left(a^{*}, b^{*}\right)$, while

- $\left(a^{*}, b^{*}\right) \succ^{*}\left(c^{*}, d^{*}\right)$ is equivalent to $\left(a^{*}, b^{*}\right) \succsim^{*}\left(c^{*}, d^{*}\right)$ and $\operatorname{not}\left[\left(c^{*}, d^{*}\right) \succsim^{*}\left(a^{*}, b^{*}\right)\right]$.

The preference information provided by the DM permits to define the following set $E^{A^{R}}$ of constraints representing the set of all the capacities compatible with the preference information given by the DM: 


$$
\begin{aligned}
& C_{\mu}\left(a^{*}\right) \geq C_{\mu}\left(b^{*}\right) \text { if } a^{*} \succsim b^{*}, \\
& C_{\mu}\left(a^{*}\right) \geq C_{\mu}\left(b^{*}\right)+\varepsilon \text { if } a^{*} \succ b^{*}, \\
& C_{\mu}\left(a^{*}\right)=C_{\mu}\left(b^{*}\right) \text { if } a^{*} \sim b^{*}, \\
& C_{\mu}\left(a^{*}\right)-C_{\mu}\left(b^{*}\right) \geq C_{\mu}\left(c^{*}\right)-C_{\mu}\left(d^{*}\right)+\varepsilon \text { if }\left(a^{*}, b^{*}\right) \succ^{*}\left(c^{*}, d^{*}\right), \\
& C_{\mu}\left(a^{*}\right)-C_{\mu}\left(b^{*}\right)=C_{\mu}\left(c^{*}\right)-C_{\mu}\left(d^{*}\right) \text { if }\left(a^{*}, b^{*}\right) \sim^{*}\left(c^{*}, d^{*}\right), \\
& \varphi(\{i\}) \geq \varphi(\{j\}) \text { if } i \succsim{ }_{1} j, \\
& \varphi(\{i\})=\varphi(\{j\}) \text { if } i \sim_{1} j, \\
& \varphi(\{i, j\}) \geq \varepsilon \text { if criteria } i \text { and } j \text { are positively interacting with } i, j \in G, \\
& \varphi(\{i, j\}) \leq-\varepsilon \text { if criteria } i \text { and } j \text { are negatively interacting with } i, j \in G, \\
& m(\{\emptyset\})=0, \quad \sum_{i \in G} m(\{i\})+\sum_{\{i, j\} \subseteq G} m(\{i, j\})=1, \\
& m(\{i\}) \geq 0, \forall i \in G, \quad\left[M C_{1}\right] \\
& m(\{i\})+\sum_{j \in T} m(\{i, j\}) \geq 0, \forall i \in G \text { and } \forall T \subseteq G \backslash\{i\}, T \neq \emptyset, \quad\left[M C_{2}\right]
\end{aligned}
$$

where $\varepsilon$ is an auxiliary variable used to transform the strict inequality constraints in weak inequality constraints. If $\varepsilon^{*}>0$, where $\varepsilon^{*}=\max \varepsilon$ subject to $E^{A^{R}}$, then there exists at least one capacity compatible with the preference information provided by the DM. If there is not any capacity compatible with the preference information provided by the DM, one can use techniques described in [49] to determine the minimal set of pieces of preference information that could be revised by the DM in order to remove the incompatibility of constraints in $E^{A^{R}}$.

Note 3.1. From the computational point of view, the previous problem is a linear programming problem composed of $|G|+\left(\begin{array}{c}|G| \\ 2\end{array}\right)+1$ variables and, in particular, one Möbius parameter $m(\{i\})$ for each criterion $i \in G$, one Möbius parameter $m(\{i, j\})$ for each pair of criteria $\{i, j\} \subseteq G^{3}$ and the variable $\varepsilon$. In the following, to simplify the notation, we shall denote by $(m, \varepsilon)$ the vector composed of the variables and we shall call it "the model".

The set $[N C]$ is composed of two equality constraints; the set $\left[M C_{1}\right]$ is composed of $|G|$ inequality constraints (one for each criterion $i \in G$ ) while the set $\left[M C_{2}\right]$ is composed of $|G| \cdot\left(2^{|G|-1}-1\right.$ ) inequality constraints $\left(2^{|G|-1}-1\right.$ for each criterion $\left.i \in G\right)$. Therefore, the number of monotonicity and normalization constraints will be equal to $2+|G| \cdot 2^{|G|-1}$. To these monotonicity and normalization constraints should be added as many equality or inequality constraints as the number of pieces of information provided by the DM. In particular, one equality constraint for each piece of indifference information (indifference between couples of alternatives or no difference between the importance of two criteria) and one inequality constraint for each piece of preference information (preference between two alternatives, intensity of preference between couples of alternatives, preference in the importance of two criteria) or for each positive or negative interaction.

In general, there could exist more than one capacity compatible with the preference information provided by the DM and to take into account all these compatible capacities, one can compute the necessary $\left(\succsim^{N}\right)$ and the possible $\left(\succsim^{P}\right)$ preference relations on the set of alternatives $A$ as described

\footnotetext{
${ }^{3}$ Let us observe that $m(\{i, j\})=m(\{j, i\})$ for each pair of criteria $\{i, j\}$ and this is the reason for which the Möbius paremeters $m(\{i, j\})$ are $\left(\begin{array}{c}|G| \\ 2\end{array}\right)$.
} 
below.

Given the following sets of constraints,

$$
\left.\left.\begin{array}{l}
C_{\mu}(b) \geq C_{\mu}(a)+\varepsilon \\
E^{A^{R}},
\end{array}\right\} E^{N}(a, b) \quad \begin{array}{l}
C_{\mu}(a) \geq C_{\mu}(b) \\
E^{A^{R}},
\end{array}\right\} E^{P}(a, b)
$$

we have that:

- $a \succsim^{N} b$ if $E^{N}(a, b)$ is infeasible or if $\varepsilon^{N} \leq 0$, where $\varepsilon^{N}=\max \varepsilon$ subject to $E^{N}(a, b)$,

- $a \succsim^{P} b$ if $E^{P}(a, b)$ is feasible and $\varepsilon^{P}>0$, where $\varepsilon^{P}=\max \varepsilon$ subject to $E^{P}(a, b)$.

Note 3.2. To get the necessary and possible preference relations one has to solve two linear programming problems in which the considered variables are the same of $E^{A^{R}}$, while the number of constraints augmented by one unity.

To check if $a \succsim^{N} b$, we add the constraint $C_{\mu}(b) \geq C_{\mu}(a)+\varepsilon$, corresponding to the strict preference of $b$ over $a$, to the set $E^{A^{R}}$ containing the constraints translating the preference information provided by the DM and the monotonicity and normalization constraints. Since we already knew that the set $E^{A^{R}}$ is feasible and that there exists at least one model $(m, \varepsilon)$ such that $\varepsilon>0$, we have the following possible cases:

- $E^{N}(a, b)$ is infeasible: this means that the strict preference of b over a is not compatible with the preference information provided by the DM since there does not exist any model $(m, \varepsilon)$ for which all the constraints are satisfied (independently from the sign of $\varepsilon$ ). Because it is not possible to find any model compatible with the preference information provided by the DM for which $b$ is strictly preferred to $a$, then a will be at least as good as $b$ for all compatible models. Consequently, $a \succsim^{N} b$.

- $E^{N}(a, b)$ is feasible but $\varepsilon^{N} \leq 0$ : in this case, even if there exist some model $(m, \varepsilon)$ for which the constraints are all satisfied, none of them has one $\varepsilon$ greater than zero. This means that for none of these models $C_{\mu}(b)>C_{\mu}(a)$ and again, $a$ is therefore at least as good as $b$ for all compatible models, implying that $a \succsim^{N} b$.

- $E^{N}(a, b)$ is feasible but $\varepsilon^{N}>0$ : in this case, there exist at least one model $(m, \varepsilon)$ for which all constraints are satisfied but, there exist at least one model presenting $\varepsilon>0$. This means that $b$ could be strictly preferred to a and, consequently, it is not true that a is at least as good as $b$ for all compatible models.

To check if $a \succsim^{P} b$, we add the constraint $C_{\mu}(a) \geq C_{\mu}(b)$, corresponding to the statement " $a$ is at least as good as b", to the set $E^{A^{R}}$. Since we already know that the set $E^{A^{R}}$ is feasible and that there exists at least one model $(m, \varepsilon)$ such that $\varepsilon>0$, we have the following possible cases:

- $E^{P}(a, b)$ is infeasible: this means that the weak preference of a over $b$ is incompatible with the preference information provided by the DM since there does not exist any model $(m, \varepsilon)$ for which all the constraints are satisfied (independently from the sign of $\varepsilon$ ). Because it is not possible to find any model compatible with the preference information provided by the DM for which a is weakly preferred to $b$, then $b$ will be strictly preferred to a for all compatible models that implies that $a \succsim^{N} b$.

- $E^{P}(a, b)$ is feasible but $\varepsilon^{P}>0$ : in this case, there exist some model $(m, \varepsilon)$ presenting $\varepsilon>0$ for which the constraints are all satisfied. This implies that there exists at least one compatible model for which $a$ is at least as good as $b$, that is $a \succsim^{P} b$. 
- $E^{P}(a, b)$ is feasible but $\varepsilon^{P} \leq 0$ : this means that there exists some model $(m, \varepsilon)$ for which all constraints are satisfied but none of these models presents an $\varepsilon>0$. Therefore, the new constraint $C_{\mu}(a) \geq C_{\mu}(b)$ is not compatible with the preference information provided by the DM and, consequently, it is not true that $a \succsim^{P} b$.

Finally, let us briefly remind that for each pair of alternatives $a, b \in A, a \succsim^{N} b$ implies $a \succsim^{P} b$ (therefore we can state that $\succsim^{N} \subseteq \succsim^{P}$ ), and that for all $a, b \in A, a \succsim^{N} b$ or $b \succsim^{P} a$ (for further important properties of the necessary and possible preference relations, see [37]). We have reminded this point to underline that to compute the necessary and possible preference relations we do not need, in general, to solve two LP problems for each couple of alternatives $(a, b)$. In particular, after computing the necessary preference relation for each couple of alternatives $(a, b)$, we need to compute only the possible preference relation for the couples $(b, a)$ such that $a \succsim^{N} b$ but not $\left(b \succsim^{N} a\right)$. Indeed, by considering the two properties mentioned above, we can have the following three cases:

- $a \succsim^{N} b$ and $b \succsim^{N} a \Rightarrow a \succsim^{P} b$ and $b \succsim^{P} a$;

- $a \succsim^{N} b$ and $\operatorname{not}\left(b \succsim^{N} a\right) \Rightarrow a \succsim^{P} b$ but nothing could be said about $b \succsim^{P} a$;

- $\operatorname{not}\left(a \succsim^{N} b\right)$ and $\operatorname{not}\left(b \succsim^{N} a\right) \Rightarrow b \succsim^{P} a$ and $a \succsim^{P} b$.

In some cases, and also in our approach, one needs to assign a real number to the overall evaluation of each alternative in order to obtain a complete ranking of the alternatives.

With this aim, based on the results of the ROR, among all the compatible models one can compute the most representative model being that one maximizing the difference in the performance of two alternatives $a$ and $b$ for which $a \succsim^{N} b$ but not $\left(b \succsim^{N} a\right)$, and minimizing the difference in the performances of two alternatives $a$ and $b$ such that $a \succsim^{P} b$ and $b \succsim^{P} a[3,22]$.

The considered procedure is composed of two steps:

$\left(S_{1}\right)$ Solving the following optimization problem:

$\max \varepsilon$ subject to

$$
\left.\begin{array}{l}
C_{\mu}(a) \geq C_{\mu}(b) \text { if } a \succsim^{N} b \text { and } \operatorname{not}\left(b \succsim^{N} a\right), \\
E^{A^{R}} .
\end{array}\right\} E_{1}
$$

$\left(S_{2}\right)$ Denoted by $\varepsilon_{1}$ the optimal value of epsilon obtained in the previous step, solving the following optimization problem:

$$
\left.\begin{array}{l}
\min \delta \text { subject to } \\
\begin{array}{l}
C_{\mu}(a)-C_{\mu}(b) \leq \delta, \\
C_{\mu}(b)-C_{\mu}(a) \leq \delta,
\end{array} \\
E_{1}, \\
\varepsilon=\varepsilon_{1} .
\end{array} \text { if } a \succsim^{P} b \text { and } b \succsim^{P} a, \quad\right\}
$$

\subsection{Analytic Hierarchy Process}

\subsubsection{Description of the method}

AHP is a multi-criteria decision making (MCDM) method that helps the DM in solving a complex problem having multiple conflicting criteria $[40,55,56]$. In its full version, AHP is structured in a hierarchy, where the decision goal is located on the top. The children of the tree are the criteria and sub-criteria to be satisfied by each alternative. 
In our paper, we are not solving the whole problem with AHP but we are only inducing a rating table. Therefore, only the core feature of AHP, the pairwise comparison matrix, is needed. The method of pairwise comparisons provides more accurate results than direct evaluations, primarily due to the fact that the DM is asked to concentrate only on two elements at a time $[18,39,48]$. With respect to MACBETH [5], another methodology to construct a scale from pairwise comparisons, AHP has the advantage to consider also indirect comparisons to derive priorities [41, 65], and this, on one hand, permits to better control the consistency of information supplied by the DM, and, on the other hand, gives more reliable results.

The pairwise comparisons are entered in a positive reciprocal matrix $\mathbf{A}=\left[a_{i j}\right]$ of dimension $n$, where $n$ is the number of considered "objects", and $a_{i j}$ expresses how many times "object" $i$ is "greater" than "object" $j$. Indeed if $p_{i}$ measures the magnitude of $i$ and $p_{j}$ the magnitude of $j$, we should have $a_{i j}=\frac{p_{i}}{p_{j}}$. Usually, following Saaty, $a_{i j}$ is determined by asking the DM a verbal judgment using the scale "moderately more dominant", "strongly more dominant", "very strongly more dominant", and "extremely more dominant" which are numerically coded as 3, 5, 7, and 9, respectively, with $2,4,6$, and 8 for compromise between the previous values. The values $a_{i j}$ from matrix $A$ are consistent if

1) $a_{i j}=\frac{1}{a_{j i}}$ for all $i$ and $j$, and this is always satisfied because, as above mentioned, matrix $\mathbf{A}$ is supposed reciprocal, and

2) $a_{i j} a_{j k}=a_{i k}$ for all $i, j$ and $k$.

It can also be written that values $a_{i j}$ from matrix $\mathbf{A}$ are consistent if and only if there exist values $p_{1}, \ldots, p_{n}$ such that for $i=1, \ldots, n$

$$
a_{i 1} p_{1}+\ldots+a_{i n} p_{n}=n \cdot p_{i}
$$

which can be written as

$$
\mathbf{A} \cdot \mathbf{p}=n \cdot \mathbf{p}
$$

where $\mathbf{p}=\left[p_{i}\right]$ is the priorities vector. In this case the priorities $p_{1}, \ldots, p_{n}$ and, consequently, the vector $\mathbf{p}$, can be easily determined as follows:

$$
p_{i}=\frac{a_{i j}}{a_{1 j}+\ldots+a_{n j}} \quad \text { for } i=1, \ldots, n .
$$

If (17) holds, (18) gives the same values for any $j=1, \ldots, n$.

However, condition (17) is rarely satisfied which implies that (18) cannot be used because it gives different values to $p_{i}$ when different $j$ are considered. Therefore several methods have been proposed to induce the priority vector $\mathbf{p}$ and among them the most well-known is the eigenvalue method calculating the priorities corresponding to matrix $\mathbf{A}$ as follows:

$$
\mathbf{A} \cdot \mathbf{p}=\lambda_{\max } \cdot \mathbf{p}
$$

where $\lambda_{\max }$ is the maximal eigenvalue of matrix $\mathbf{A}$.

In order to declare the comparison matrix consistent enough for calculating credible priorities, it must pass a consistency check. Consistency Ratio (CR) is defined as:

$$
C R=C I / R I,
$$

where RI is the Random Index (the average CI of 500 randomly filled matrices).

$\mathrm{CI}$ is the Consistency Index 


$$
C I=\left(\lambda_{\max }-n\right) /(n-1) .
$$

In order to make the rating on different criteria commensurable, we normalise the priorities with respect to the maximum and minimum score on each criterion as follows:

$$
p_{i}^{*}=\frac{p_{i}-p_{\min }}{p_{\max }-p_{\min }}
$$

where:

- $p_{i}^{*}$ is the rating of the score $i$,

- $p_{i}$ is the calculated priority of the score $i$,

- $p_{\max }$ is the priority of the maximum score,

- $p_{\min }$ is the priority of the minimum score.

Observe that the rating of $p_{i}^{*}$ will be in the range $[0,1]$, and $p_{i}^{*}=1$ if $p_{i}=p_{\max }$ and $p_{i}^{*}=0$ if $p_{i}=p_{\min }$.

\subsubsection{Reducing the number of pairwise comparisons}

AHP permits to build a scale on each criterion starting from ratio evaluations given by the DM. This is very important in multiple criteria decision making because numerical evaluations of alternatives on considered criteria permit to proceed towards their aggregation in a single overall evaluation allowing to compare comprehensively alternatives between them. However, the application of the AHP becomes troublesome when the number of considered alternatives is high. Indeed, if the number of alternatives is $m$, then there is the necessity of $\frac{m(m-1)}{2}$ pairwise comparisons for each considered criterion, such that, for $n$ criteria we have a total of $n \frac{m(m-1)}{2}$ pairwise comparisons to ask to the DM. For example, for a not so complex problem with 7 criteria and 7 alternatives, the total number of pairwise comparisons to ask to the DM is 147, which becomes 450 in a problem with 10 criteria and 10 alternatives and 1900 in a problem with 10 criteria and 20 alternatives. Thus the use of AHP requires a consistent cognitive efforts from the DM and this can deteriorate the quality of the information provided with the risk that the MCDA methodology gives back a result which is not enough reliable. Consider also that, sometimes, there is the necessity to get a decision model that can be applied to a set of alternatives very large and not predefined at the beginning. For instance, suppose that one wants to develop a model to assess credit score for customers of a bank taking into account several financial ratios such as return on equity, current ratio, debt ratio and so on. In this case, it is necessary to build a decision model which is universally applicable to all the customers requiring a credit to the bank. Thus, we should consider thousands of potential customers and, even if we would be able to get the billions of pairwise comparisons necessary to apply AHP, in any case, the customers that could apply for a credit cannot be known in advance. Therefore, there is the necessity to develop a method permitting to apply AHP also in these cases. For this reason, we propose to fix a small number of representative points in the scale of each criterion and to ask the DM to compare pairwise these points. After obtaining through AHP a normalized evaluation for these points, the evaluations of all other alternatives with respect to the considered criteria can be obtained by linear interpolation. More formally, supposing for the sake of simplicity that all criteria have a numerical scale that is monotonic increasing with respect to the preferences, for each criterion $i, i=1, \ldots, n$, we consider $t_{i}$ representative points well distributed on the scale of the criterion. Let us denote by $\gamma_{i r}$ the $r$-th point, $r=1, \ldots, t_{i}$, on the scale of criterion $i$. For each criterion $i$, the DM is asked to supply $t_{i} \frac{t_{i}-1}{2}$ pairwise comparisons between points $\gamma_{i r}$ and $\gamma_{i s}, r, s=1, \ldots, t_{i}$. Using AHP, 
for each representative point $\gamma_{i r}$, the normalized evaluations $u\left(\gamma_{i r}\right)$ are obtained. Consider now an alternative $a$ having an evaluation $g_{i}(a)$ on criterion $i$. If $g_{i}(a)$ belongs to the interval of consecutive representative points $\left[\gamma_{i s}, \gamma_{i(s+1)}\right]$, we can get the normalized evaluation $u\left(g_{i}(a)\right)$ of $g_{i}(a)$ as follows:

$$
u\left(g_{i}(a)\right)=u\left(\gamma_{i s}\right)+\frac{u\left(\gamma_{i(s+1)}\right)-u\left(\gamma_{i s}\right)}{\gamma_{i(s+1)}-\gamma_{i s}}\left(g_{i}(a)-\gamma_{i s}\right) .
$$

It is to observe that the selected set of points may have an influence on the results of the rescaling. They must therefore be selected carefully and in the most representative way. We believe that the best way to select reference points is to select them in cooperation with the DM. Indeed, the fundamental characteristic that the reference points must possess is their meaningfulness for the DM. In addition to the greater reliability of her comparisons, there is another advantage in involving the DM in the selection of the reference points. Indeed, the more the reference points are meaningful for the DM, the more she will feel comfortable in reasoning about possible inconsistencies and correct them, if necessary.

With respect to the procedure we are proposing, one could ask if the reduction of comparisons does not reduce the possibility of discovering inconsistencies in the DM's evaluations, with the risk of obtaining a decision model not enough accurate and convincing. We do not believe that this is the case. Indeed, asking for comparing all the alternatives, especially in case of a great number of alternatives, the DM experiments a great cognitive burden which is not compensated by a greater reliability of the results. It is rather the contrary. The greater the number of the questions requested to the DM and the more difficult the contents of these questions (because very often are based on negligible differences), the less is the attention in answering and the more deteriorate the overall quality of the preference information collected by the DM. Let us observe that this consideration is confirmed by the growing literature in the domain of the heuristic decision making (see e.g. [28]) which is mainly based on the principle that ignoring part of the information can lead to more accurate and more effective decisions. Moreover, even supposing an "ideal" DM able to answer in a good way to all the many questions one can ask her, the traditional AHP is not adapted for large problems. Observe that some studies even recommend only 7 alternatives because of the limited capacity of our brain to compare more alternatives [59]. Therefore, reducing reasonably the number of pairwise comparisons will not reduce the quality of the obtained decision model because the traditional AHP will anyway have a reduced quality with a high number of alternatives and, probably, it will also increase the reliability of the final results.

Since AHP has received several criticisms, one could wonder how they apply to the methodology we are proposing. We believe that the soundness of our application of AHP is not touched by these remarks. For example, we agree with Salo and Hämäläinen [60] that the 1-9 scale is not always justified. However, recent publications $[43,52]$ have proposed to calibrate the measurement scale to the mental representation of the DM. We have not done this extra step because it is not the main focus of the paper, but it can easily be added. Also the criticism of Perez, Jimeno and Mokotoff [51] regarding the use of indifferent criteria in AHP does not apply to our model. Indeed, in our work, we only use partially AHP. More precisely, we use only the pairwise comparison matrices for comparing performances but we do not use pairwise comparison matrices for finding the weights of the criteria since we use the NAROR technique for this part. Bana e Costa and Vansnick [6] observe that the Condition of Order Preservation, that is, loosely speaking, that priorities represent also intensity of preferences between alternatives, is not preserved in AHP. This is true if we consider direct evaluations only. As we consider useful to take into account direct and indirect evaluations to derive priorities $[41,65]$, also this criticism is not applicable to our model.

Finally, it is important to mention that the procedure we are proposing consisting of compare pairwise a small number of reference points only can also be applied to other methods that give a normalized evaluation on the basis of pairwise comparisons as, for example, MACBETH [5]. 


\section{An application for the conjoint use of NAROR and AHP}

Steve wants to buy an economy car and, therefore, he is analyzing currently available cars on the market. Thus, he decides to consider 24 models that are presented in Table 3 together with their evaluations with respect to criteria Price, Acceleration, Max Speed and Fuel Consumption. First of all, in order to use the Choquet integral preference model and the NAROR to decide which car to buy, the evaluations of each car with respect to above criteria have to be expressed on a common scale. This is possible using AHP but it requires 276 pairwise comparisons for each one of the considered criteria which lead to a total of 1104 pairwise comparisons. Steve thinks that all these pairwise comparisons are too many, and thus we propose him to apply AHP only to a set of representative evaluations on the scale of each criterion and to determine the normalized value of the evaluations of the 24 considered cars by using the procedure described in Section 3.4.2.

Table 3: Set of considered cars and their evaluation

\begin{tabular}{|c|c|c|c|c|}
\hline & Price [Euro] & Acceleration [seconds from 0 to $100 \mathrm{~km} / \mathrm{h}$ ] & Max Speed $[\mathrm{km} / \mathrm{h}]$ & Consumption $(\mathrm{l} / \mathrm{km})$ \\
\hline (a $\left.a_{1}\right)$ Audi A3 (3-doors) & 22,140 & 10.3 & 193 & 4.9 \\
\hline$\left(a_{2}\right)$ BMW 1 Series (3-doors) & 23,089 & 11.2 & 195 & 5.5 \\
\hline$\left(a_{3}\right)$ Hyunday ix20 & 14,000 & 12.9 & 167 & 6 \\
\hline$\left(a_{4}\right)$ Ford C-Max & 19,500 & 12.6 & 174 & 5.1 \\
\hline$\left(a_{5}\right)$ Toyota Aygo & 10,350 & 13.7 & 157 & 4.4 \\
\hline ( $\left.a_{6}\right)$ Seat Ibiza (5-doors) Style & 13,000 & 13.9 & 163 & 5.4 \\
\hline$\left(a_{7}\right)$ VolksWagen Polo highline 1.4 (3-door) & 16,550 & 12.1 & 177 & 5.9 \\
\hline (a) BMW Serie 1 (3-doors) & 23,069 & 11.2 & 195 & 5.5 \\
\hline$\left(a_{9}\right)$ Chevrolet Spark & 9,952 & 15.3 & 152 & 5 \\
\hline$\left(a_{10}\right)$ FIAT Punto (3-doors) & 13,711 & 11.2 & 182 & 4.2 \\
\hline$\left(a_{11}\right)$ Ford Fiesta (3-doors) & 12,750 & 14.9 & 165 & 4.6 \\
\hline$\left(a_{12}\right)$ Honda Civic & 18,900 & 13.4 & 187 & 5.4 \\
\hline$\left(a_{13}\right)$ Kia Rio & 11,650 & 13.1 & 172 & 5.1 \\
\hline$\left(a_{14}\right)$ Lancia Ypsilon & 14,568 & 11.9 & 176 & 4.2 \\
\hline$\left(a_{15}\right)$ Mazda2 3-door Sporty & 14,900 & 13.6 & 172 & 5 \\
\hline$\left(a_{16}\right)$ Mercedes A-Class & 23,630 & 9.2 & 202 & 5.5 \\
\hline$\left(a_{17}\right)$ Mini Cooper & 20,700 & 7.9 & 210 & 4.5 \\
\hline$\left(a_{18}\right)$ Mitsubishi Space Star & 11,490 & 13.6 & 172 & 4 \\
\hline$\left(a_{19}\right)$ Nissan Micra & 11,250 & 13.7 & 170 & 5 \\
\hline$\left(a_{20}\right)$ Opel Corsa & 11,330 & 18.2 & 155 & 5.1 \\
\hline$\left(a_{21}\right)$ Peugeot 208 & 12,100 & 14 & 163 & 4.3 \\
\hline$\left(a_{22}\right)$ Renault Clio & 16,200 & 12.2 & 182 & 4.5 \\
\hline$\left(a_{23}\right)$ Skoda Citygo & 9,260 & 14.4 & 160 & 4.5 \\
\hline$\left(a_{24}\right)$ Suzuki Swift & 12,100 & 11.5 & 165 & 5 \\
\hline
\end{tabular}

For this reason, we ask Steve to compare the representative values shown in Table 4. As a consequence, the pairwise comparisons asked to Steve are:

- 55 for the 11 reference levels of criterion Price,

- 28 for the 8 reference levels of criterion Acceleration,

- 21 for the 7 reference levels of criterion Max Speed,

- 36 for the 9 reference levels of criterion Consumption,

which gives a total of 140 pairwise comparisons. The pairwise comparisons given by Steve are shown in Tables 5(a)-5(d) while the normalized evaluations of these reference points, obtained by AHP, are provided in Table 6 . Considering the normalized evaluations of the reference points and interpolating them as described in the previous Section, we are able to obtain the normalized evaluations of all cars with respect to all criteria reported in Table 7. For example, to obtain the normalized evaluation of the Kia Rio with respect to price, first of all we have to observe that its price (11,650 euro), is in the interval of references prices whose extremes are 10,500 euro and 12,000 euro. Since the utilities 
of these reference prices obtained by AHP are respectively 0.2907 and 0.2489 , applying (19) we get the normalized price of the Kia Rio as follows:

$$
\begin{aligned}
u(11,650) & =u(10,500)+\frac{u(12,000)-u(10,500)}{12,000-10,500}(11,650-10,500) \\
& =0.2907+\frac{0.2489-0.2907}{1,500}(1,150)=0.2587
\end{aligned}
$$

Table 4: Reference levels for considered criteria

\begin{tabular}{|c|c|c|c|}
\hline Price [Euro] & Acceleration [seconds from 0 to $\mathbf{1 0 0} \mathbf{~ k m} / \mathbf{h}]$ & Max Speed $[\mathbf{K m} / \mathbf{h}]$ & Consumption $(\mathbf{l} / \mathbf{k m})$ \\
\hline 9,000 & 7 & 150 & 4 \\
10,500 & 9 & 160 & 4.25 \\
12,000 & 10.5 & 170 & 4.5 \\
13,500 & 12 & 180 & 4.75 \\
15,000 & 13.5 & 190 & 5 \\
17,000 & 15 & 200 & 5.25 \\
19,000 & 16.5 & 210 & 5.5 \\
21,000 & 19 & & 6.75 \\
23,000 & & & \\
25,000 & & & \\
\hline
\end{tabular}

Table 5: Pairwise comparison matrix for the considered criteria

(a) Price $(\mathrm{CI}=0.1)$

\begin{tabular}{|r|c|c|c|c|c|c|c|c|c|c|}
\hline & $\mathbf{9 , 0 0 0}$ & $\mathbf{1 0 , 5 0 0}$ & $\mathbf{1 2 , 0 0 0}$ & $\mathbf{1 3 , 5 0 0}$ & $\mathbf{1 5 , 0 0 0}$ & $\mathbf{1 7 , 0 0 0}$ & $\mathbf{1 9 , 0 0 0}$ & $\mathbf{2 1 , 0 0 0}$ & $\mathbf{2 3 , 0 0 0}$ & $\mathbf{2 5 , 0 0 0}$ \\
\hline $\mathbf{9 , 0 0 0}$ & 1 & 9 & 9 & 9 & 9 & 9 & 9 & 9 & 9 & 9 \\
$\mathbf{1 0 , 5 0 0}$ & $1 / 9$ & 1 & 2 & 3 & 3 & 4 & 6 & 7 & 9 & 9 \\
$\mathbf{1 2 , 0 0 0}$ & $1 / 9$ & $1 / 2$ & 1 & 2 & 3 & 5 & 6 & 8 & 9 & 9 \\
$\mathbf{1 3 , 5 0 0}$ & $1 / 9$ & $1 / 3$ & $1 / 2$ & 1 & 2 & 3 & 3 & 4 & 6 & 9 \\
$\mathbf{1 5 5 0 0 0}$ & $1 / 9$ & $1 / 3$ & $1 / 3$ & $1 / 2$ & 1 & 2 & 4 & 4 & 6 & 9 \\
$\mathbf{1 7 , 0 0 0}$ & $1 / 9$ & $1 / 4$ & $1 / 5$ & $1 / 3$ & $1 / 2$ & 1 & 2 & 3 & 4 & 6 \\
$\mathbf{1 9 , 0 0 0}$ & $1 / 9$ & $1 / 6$ & $1 / 6$ & $1 / 3$ & $1 / 4$ & $1 / 2$ & 1 & 2 & 3 & 5 \\
$\mathbf{2 1 , 0 0 0}$ & $1 / 9$ & $1 / 7$ & $1 / 8$ & $1 / 4$ & $1 / 4$ & $1 / 3$ & $1 / 2$ & 1 & 2 & 4 \\
$\mathbf{2 3 , 0 0 0}$ & $1 / 9$ & $1 / 9$ & $1 / 9$ & $1 / 6$ & $1 / 6$ & $1 / 4$ & $1 / 3$ & $1 / 2$ & 1 & 2 \\
$\mathbf{2 5 , 0 0 0}$ & $1 / 9$ & $1 / 9$ & $1 / 9$ & $1 / 9$ & $1 / 9$ & $1 / 6$ & $1 / 5$ & $1 / 4$ & $1 / 2$ & 1 \\
\hline
\end{tabular}

(c) Speed $(\mathrm{CI}=0.1)$

\begin{tabular}{|c|c|c|c|c|c|c|c|}
\hline & $\mathbf{1 5 0}$ & $\mathbf{1 6 0}$ & $\mathbf{1 7 0}$ & $\mathbf{1 8 0}$ & $\mathbf{1 9 0}$ & $\mathbf{2 0 0}$ & $\mathbf{2 1 0}$ \\
\hline $\mathbf{1 5 0}$ & 1 & $1 / 5$ & $1 / 6$ & $1 / 7$ & $1 / 8$ & $1 / 8$ & $1 / 8$ \\
$\mathbf{1 6 0}$ & 5 & 1 & $1 / 2$ & $1 / 6$ & $1 / 7$ & $1 / 7$ & $1 / 7$ \\
$\mathbf{1 7 0}$ & 6 & 2 & 1 & $1 / 2$ & $1 / 6$ & $1 / 7$ & $1 / 7$ \\
$\mathbf{1 8 0}$ & 7 & 6 & 2 & 1 & $1 / 5$ & $1 / 5$ & $1 / 5$ \\
$\mathbf{1 9 0}$ & 8 & 7 & 6 & 5 & 1 & $1 / 2$ & $1 / 3$ \\
$\mathbf{2 0 0}$ & 8 & 7 & 7 & 5 & 2 & 1 & 1 \\
$\mathbf{2 1 0}$ & 8 & 7 & 7 & 5 & 3 & 1 & 1 \\
\hline
\end{tabular}

(b) Acceleration $(\mathrm{CI}=0.05)$

\begin{tabular}{|r|c|c|c|c|c|c|c|c|}
\hline & $\mathbf{7}$ & $\mathbf{9}$ & $\mathbf{1 0 . 5}$ & $\mathbf{1 2}$ & $\mathbf{1 3 . 5}$ & $\mathbf{1 5}$ & $\mathbf{1 6 . 5}$ & $\mathbf{1 9}$ \\
\hline $\mathbf{7}$ & 1 & 3 & 4 & 6 & 7 & 7 & 8 & 9 \\
$\mathbf{9}$ & $1 / 3$ & 1 & 2 & 4 & 5 & 6 & 7 & 9 \\
$\mathbf{1 0 . 5}$ & $1 / 4$ & $1 / 2$ & 1 & 2 & 4 & 5 & 6 & 8 \\
$\mathbf{1 2}$ & $1 / 6$ & $1 / 4$ & $1 / 2$ & 1 & 2 & 3 & 4 & 6 \\
$\mathbf{1 3 . 5}$ & $1 / 7$ & $1 / 5$ & $1 / 4$ & $1 / 2$ & 1 & 2 & 3 & 5 \\
$\mathbf{1 5}$ & $1 / 7$ & $1 / 6$ & $1 / 5$ & $1 / 3$ & $1 / 2$ & 1 & 2 & 4 \\
$\mathbf{1 6 . 5}$ & $1 / 8$ & $1 / 7$ & $1 / 6$ & $1 / 4$ & $1 / 3$ & $1 / 2$ & 1 & 2 \\
$\mathbf{1 9}$ & $1 / 9$ & $1 / 9$ & $1 / 8$ & $1 / 6$ & $1 / 5$ & $1 / 4$ & $1 / 2$ & 1 \\
\hline
\end{tabular}

(d) Fuel consumption $(\mathrm{CI}=0.08)$

\begin{tabular}{|r|c|c|c|c|c|c|c|c|c|}
\hline & $\mathbf{4}$ & $\mathbf{4 . 2 5}$ & $\mathbf{4 . 5}$ & $\mathbf{4 . 7 5}$ & $\mathbf{5}$ & $\mathbf{5 . 2 5}$ & $\mathbf{5 . 5}$ & $\mathbf{5 . 7 5}$ & $\mathbf{6}$ \\
\hline $\mathbf{4}$ & 1 & 3 & 4 & 5 & 6 & 7 & 8 & 9 & 9 \\
$\mathbf{4 . 2 5}$ & $1 / 3$ & 1 & 2 & 4 & 5 & 6 & 7 & 8 & 9 \\
$\mathbf{4 . 5}$ & $1 / 4$ & $1 / 2$ & 1 & 2 & 3 & 5 & 6 & 8 & 9 \\
$\mathbf{4 . 7 5}$ & $1 / 5$ & $1 / 4$ & $1 / 2$ & 1 & 2 & 4 & 5 & 6 & 7 \\
$\mathbf{5}$ & $1 / 6$ & $1 / 5$ & $1 / 3$ & $1 / 2$ & 1 & 2 & 4 & 5 & 7 \\
$\mathbf{5 . 2 5}$ & $1 / 7$ & $1 / 6$ & $1 / 5$ & $1 / 4$ & $1 / 2$ & 1 & 2 & 4 & 6 \\
$\mathbf{5 . 5}$ & $1 / 8$ & $1 / 7$ & $1 / 6$ & $1 / 5$ & $1 / 4$ & $1 / 2$ & 1 & 2 & 7 \\
$\mathbf{5 . 7 5}$ & $1 / 9$ & $1 / 8$ & $1 / 8$ & $1 / 6$ & $1 / 5$ & $1 / 4$ & $1 / 2$ & 1 & 3 \\
$\mathbf{6}$ & $1 / 9$ & $1 / 9$ & $1 / 9$ & $1 / 7$ & $1 / 7$ & $1 / 6$ & $1 / 7$ & $1 / 3$ & 1 \\
\hline
\end{tabular}

To apply the Choquet integral preference model, we decide to use the indirect preference information and, consequently, Steve provides some preference information about the interaction between criteria (the constraints translating the corresponding information are in brackets):

- Price $(\mathrm{P})$ and Acceleration $(\mathrm{A})$ are positively interacting $(\varphi(P, A) \geq \varepsilon)$,

- Price and Max Speed (M) are positively interacting $(\varphi(P, M) \geq \varepsilon)$, 
Table 6: Reference levels for considered criteria and corresponding normalized values obtained by AHP

\begin{tabular}{|c|c|c|c|c|c|c|c|}
\hline Price [Euro] & Norm. & Acceleration [seconds from 0 to $\mathbf{1 0 0} \mathbf{~ k m} / \mathbf{h}$ ] & Norm. & Max Speed [Km/h] & Norm. & Consumption (l/km) & Norm. \\
\hline 9,000 & 1 & 7 & 1 & 150 & 0 & 4 & 1 \\
10,500 & 0.2907 & 9 & 0.5595 & 160 & 0.0616 & 4.25 & 0.6224 \\
12,000 & 0.2489 & 10.5 & 0.3622 & 170 & 0.1096 & 4.5 & 0.4079 \\
13,500 & 0.1454 & 12 & 0.1892 & 180 & 0.2432 & 4.75 & 0.2659 \\
15,000 & 0.1167 & 13.5 & 0.1108 & 190 & 0.6438 & 5 & 0.1692 \\
17,000 & 0.0661 & 16.5 & 0.0649 & 200 & 0.9110 & 5.25 & 0.0967 \\
19,000 & 0.0396 & 19 & 0.0243 & 210 & 1 & 5.5 & 0.0574 \\
21,000 & 0.0220 & & & & & & 5.75 \\
23,000 & 0.0088 & & & & & & 0.0211 \\
25,000 & 0 & & & & & & \\
\hline
\end{tabular}

Table 7: Set of considered cars with normalized evaluations on each criterion

\begin{tabular}{|c|c|c|c|c|}
\hline & Price [Euro] & Acceleration [seconds from 0 to $100 \mathrm{~km} / \mathrm{h}$ ] & Max Speed $[\mathrm{Km} / \mathrm{h}]$ & Consumption (l/km) \\
\hline$\left(a_{1}\right)$ Audi A3 (3-doors) & 0.0145 & 0.3885 & 0.7240 & 0.2079 \\
\hline$\left(a_{2}\right)$ BMW 1 Series (3-doors) & 0.0084 & 0.2814 & 0.7774 & 0.0574 \\
\hline$\left(a_{3}\right)$ Hyunday ix20 & 0.1358 & 0.1422 & 0.0952 & 0.0000 \\
\hline$\left(a_{4}\right)$ Ford C-Max & 0.0352 & 0.1578 & 0.1630 & 0.1402 \\
\hline$\left(a_{6}\right)$ Seat Ibiza (5-doors) Style & 0.1799 & 0.0986 & 0.0760 & 0.0731 \\
\hline$\left(a_{7}\right)$ VolksWagen Polo highline 1.4 (3-door) & 0.0775 & 0.1840 & 0.2031 & 0.0085 \\
\hline (as $)$ BMW Serie 1 (3-doors) & 0.0085 & 0.2814 & 0.7774 & 0.0574 \\
\hline$\left(a_{9}\right)$ Chevrolet Spark & 0.5499 & 0.0568 & 0.0123 & 0.1692 \\
\hline$\left(a_{11}\right)$ Ford Fiesta (3-doors) & 0.1971 & 0.0679 & 0.0856 & 0.3511 \\
\hline$\left(a_{12}\right)$ Honda Civic & 0.0410 & 0.1160 & 0.5236 & 0.0731 \\
\hline$\left(a_{13}\right)$ Kia Rio & 0.2587 & 0.1317 & 0.1363 & 0.1402 \\
\hline$\left(a_{14}\right)$ Lancia Ypsilon & 0.1250 & 0.2007 & 0.1897 & 0.6979 \\
\hline$\left(a_{15}\right)$ Mazda2 3-door Sporty & 0.1186 & 0.1077 & 0.1363 & 0.1692 \\
\hline$\left(a_{16}\right)$ Mercedes A-Class & 0.0060 & 0.5332 & 0.9288 & 0.0574 \\
\hline$\left(a_{17}\right)$ Mini Cooper & 0.0247 & 0.8018 & 1.0000 & 0.4079 \\
\hline$\left(a_{18}\right)$ Mitsubishi Space Star & 0.2631 & 0.1077 & 0.1363 & 1.0000 \\
\hline$\left(a_{19}\right)$ Nissan Micra & 0.2698 & 0.1047 & 0.1096 & 0.1692 \\
\hline$\left(a_{21}\right)$ Peugeot 208 & 0.2420 & 0.0955 & 0.0760 & 0.5795 \\
\hline$\left(a_{22}\right)$ Renault Clio & 0.0863 & 0.1787 & 0.3233 & 0.4079 \\
\hline$\left(a_{23}\right)$ Skoda Citygo & 0.8771 & 0.0832 & 0.0616 & 0.4079 \\
\hline$\left(a_{24}\right)$ Suzuki Swift & 0.2420 & 0.2468 & 0.0856 & 0.1692 \\
\hline
\end{tabular}

- Acceleration and Consumption $(\mathrm{C})$ are positively interacting $(\varphi(A, C) \geq \varepsilon)$,

- Max Speed and Consumption are positively interacting $(\varphi(M, C) \geq \varepsilon)$,

- Price and Consumption are negatively interacting $(\varphi(P, C) \leq-\varepsilon)$,

- Acceleration and Max Speed are negatively interacting $(\varphi(A, M) \leq-\varepsilon)$.

Steve provides also the following preference order on some cars that he knows and for which he is able to form his preferences:

$$
a_{3} \succ a_{5} \succ a_{6} \succ a_{7} \succ a_{1} \succ a_{4} \succ a_{2} .
$$

This preference information is translated by the following linear inequalities:

$$
\begin{array}{lll}
\text { - } C_{\mu}\left(a_{3}\right) \geq C_{\mu}\left(a_{5}\right)+\varepsilon, & C_{\mu}\left(a_{5}\right) \geq C_{\mu}\left(a_{6}\right)+\varepsilon, & C_{\mu}\left(a_{6}\right) \geq C_{\mu}\left(a_{7}\right)+\varepsilon, \\
\text { - } C_{\mu}\left(a_{7}\right) \geq C_{\mu}\left(a_{1}\right)+\varepsilon, & C_{\mu}\left(a_{1}\right) \geq C_{\mu}\left(a_{4}\right)+\varepsilon, & C_{\mu}\left(a_{4}\right) \geq C_{\mu}\left(a_{2}\right)+\varepsilon,
\end{array}
$$

where $\varepsilon$ is an auxiliary variable supposed being greater than zero.

To check if there exist at least one capacity compatible with the preferences Steve provided us, we have to solve the following linear programming problem: 


$$
\begin{aligned}
& \varepsilon^{*}=\max \varepsilon, \text { s.t. } \\
& C_{\mu}\left(a_{3}\right) \geq C_{\mu}\left(a_{5}\right)+\varepsilon, \quad C_{\mu}\left(a_{5}\right) \geq C_{\mu}\left(a_{6}\right)+\varepsilon, \\
& C_{\mu}\left(a_{6}\right) \geq C_{\mu}\left(a_{7}\right)+\varepsilon, \quad C_{\mu}\left(a_{7}\right) \geq C_{\mu}\left(a_{1}\right)+\varepsilon, \\
& C_{\mu}\left(a_{1}\right) \geq C_{\mu}\left(a_{4}\right)+\varepsilon, \quad C_{\mu}\left(a_{4}\right) \geq C_{\mu}\left(a_{2}\right)+\varepsilon, \\
& m(\{P, A\}) \geq \varepsilon, \quad m(\{P, M\}) \geq \varepsilon, \\
& m(\{A, C\}) \geq \varepsilon, \quad m(\{M, C\}) \geq \varepsilon, \\
& m(\{P, C\}) \leq-\varepsilon, \quad m(\{A, M\}) \leq-\varepsilon, \\
& m(\{\emptyset\})=0, \quad \sum_{i \in\{P, A, M, C\}} m(\{i\})+\sum_{\{i, j\} \subseteq\{P, A, M, C\}} m(\{i, j\})=1 \text {, } \\
& m(\{i\}) \geq 0, \forall i \in\{P, A, M, C\}, \\
& m(\{i\})+\sum_{j \in T} m(\{i, j\}) \geq 0, \forall i \in\{P, A, M, C\} \text { and } \forall T \subseteq\{P, A, M, C\} \backslash\{i\}, T \neq \emptyset,
\end{aligned}
$$

Because $\varepsilon^{*}$ is greater than zero, this means that there exists at least one capacity compatible with the preference information provided by Steve. Table 8 presents the Möbius representation of the capacity corresponding to $\varepsilon^{*}$.

Table 8: Möbius representation of the capacity maximizing the value of $\varepsilon$

\begin{tabular}{|cccccccccc|}
\hline $\mathbf{m}(\{P\})$ & $\mathbf{m}(\{A\})$ & $\mathbf{m}(\{M\})$ & $\mathbf{m}(\{C\})$ & $\mathbf{m}(\{P, A\})$ & $\mathbf{m}(\{P, M\})$ & $\mathbf{m}(\{P, C\})$ & $\mathbf{m}(\{A, M\})$ & $\mathbf{m}(\{A, C\})$ & $\mathbf{m}(\{M, C\})$ \\
\hline 0.0380 & 0.0833 & 0.0133 & 0.0380 & 0.7374 & 0.0809 & -0.0380 & -0.0133 & 0.0133 & 0.0472 \\
\hline
\end{tabular}

On the basis of the Möbius representation of the capacity maximizing $\varepsilon$, we can compute the Shapley index of each criterion. For example, the Shapley index of criterion price will be obtained as follows:

$\varphi(\{P\})=m(\{P\})+\frac{m(\{P, A\})+m(\{P, M\})+m(\{P, C\})}{2}=0.0380+\frac{0.7374+0.0809-0.0380}{2}=0.4281$.

Looking at Tables 8 and 9, one can see that Acceleration is the most important criterion both considered singularly (because $m(\{M\})>m(\{i\}), i \in\{P, A, C\}$ ), and also when taking into account its interactions with the other three criteria (because $\varphi(\{M\})>\varphi(\{i\}), i \in\{P, A, C\}$ ). On the other hand, Maximum Speed is the less important criterion if it is considered singularly, while Consumption is the less important criterion considering also all its interactions with the other criteria.

Table 9: Importance of criteria measured by the Shapley index

\begin{tabular}{|c|c|}
\hline & $\varphi$ \\
\hline$\{\mathbf{P}\}$ & 0.4281 \\
$\{\mathbf{A}\}$ & 0.452 \\
$\{\mathbf{M}\}$ & 0.0707 \\
$\{\mathbf{C}\}$ & 0.0492 \\
\hline
\end{tabular}

In order to take into account not only one but the whole set of capacities compatible with the preference information provided by Steve, we apply the NAROR as described in Section 3.3. In Table 
10, we reported the necessary preference relation $\succsim^{N}$, while in Table 11 we reported the asymmetric part $\left(\succ^{P}\right)$ of the possible preference relation $\succsim^{P}$, where $a \succ^{P} b$ iff $a \succsim^{P} b$ and not $\left(b \succsim^{P} a\right)$. We presented $\succ^{P}$ instead of $\succsim^{P}$, because $\succ^{P} \subseteq \succsim^{N} \subseteq \succsim^{P}$ (that is $\succ^{P}$ is much more synthetic than $\succsim^{P}$ ) and, overall, because $\succ^{P}$ is transitive, which is not the case for $\succsim^{P}$ [37]. In simple words, $\succ^{P}$ is much more intelligible than $\succsim^{P}$ for the DM.

Table 10: Necessary preference relation: for each line, the car on the left is necessarily preferred to the cars on the right. For example, $a_{1}$ is necessarily preferred to $a_{2}, a_{4}$ and $a_{8}$ while the viceversa is not true.

\begin{tabular}{|c|c|}
\hline & Cars \\
\hline $\begin{array}{c}\left(a_{1}\right) \text { Audi A3 (3-doors) } \\
\left(a_{2}\right) \text { BMW } 1 \text { Series (3-doors) } \\
\left(a_{3}\right) \text { Hyunday ix20 } \\
\left(a_{4}\right) \text { Ford C-Max } \\
\left(a_{5}\right) \text { Toyota Aygo } \\
\left(a_{6}\right) \text { Seat Ibiza (5-doors) Style } \\
\left(a_{7}\right) \text { VolksWagen Polo highline 1.4 (3-door) } \\
\left(a_{8}\right) \text { BMW Serie 1 (3-doors) } \\
\left(a_{9}\right) \text { Chevrolet Spark } \\
\left(a_{10}\right) \text { FIAT Punto (3-doors) } \\
\left(a_{11}\right) \text { Ford Fiesta (3-doors) } \\
\left(a_{12}\right) \text { Honda Civic } \\
\left(a_{13}\right) \text { Kia Rio } \\
\left(a_{14}\right) \text { Lancia Ypsilon } \\
\left(a_{15}\right) \text { Mazda2 3-door Sporty } \\
\left(a_{16}\right) \text { Mercedes A-Class } \\
\left(a_{17}\right) \text { Mini Cooper } \\
\left(a_{18}\right) \text { Mitsubishi Space Star } \\
\left(a_{19}\right) \text { Nissan Micra } \\
\left(a_{20}\right) \text { Opel Corsa } \\
\left(a_{21}\right) \text { Peugeot 208 } \\
\left(a_{22}\right) \text { Renault Clio } \\
\left(a_{23}\right) \text { Skoda Citygo } \\
\left(a_{24}\right) \text { Suzuki Swift }\end{array}$ & $\begin{array}{c}a_{2}, a_{4}, a_{8}, \\
\emptyset \\
a_{1}, a_{2}, a_{4}, a_{5}, a_{6}, a_{7}, a_{8}, a_{12}, a_{20}, \\
a_{2}, \\
a_{1}, a_{2}, a_{4}, a_{6}, a_{7}, a_{8}, a_{12}, a_{20}, \\
a_{1}, a_{2}, a_{4}, a_{7}, a_{8}, a_{12}, a_{20}, \\
a_{1}, a_{2}, a_{4}, a_{8}, a_{12}, a_{20}, \\
a_{2} \\
\emptyset \\
a_{1}, a_{2}, a_{3}, a_{4}, a_{5}, a_{6}, a_{7}, a_{8}, a_{9}, a_{11}, a_{12}, a_{14}, a_{15}, a_{16}, a_{19}, a_{20}, a_{21}, a_{22}, \\
a_{2}, a_{4}, a_{8}, a_{20}, \\
\emptyset \\
a_{1}, a_{2}, a_{4}, a_{5}, a_{6}, a_{7}, a_{8}, a_{9}, a_{11}, a_{12}, a_{15}, a_{16}, a_{19}, a_{20}, a_{21} \\
a_{1}, a_{2}, a_{4}, a_{6}, a_{7}, a_{8}, a_{9}, a_{11}, a_{12}, a_{13}, a_{15}, a_{16}, a_{20}, a_{21} \\
a_{1}, a_{2}, a_{4}, a_{6}, a_{7}, a_{8}, a_{11}, a_{12}, a_{20} \\
\emptyset \\
a_{1}, a_{2}, a_{4}, a_{8}, a_{12}, a_{16} \\
a_{1}, a_{2}, a_{4}, a_{6}, a_{7}, a_{8}, a_{9}, a_{11}, a_{12}, a_{15}, a_{19}, a_{20}, a_{21} \\
a_{1}, a_{2}, a_{4}, a_{6}, a_{7}, a_{8}, a_{9}, a_{11}, a_{12}, a_{20} \\
\emptyset \\
a_{2}, a_{4}, a_{8}, a_{12}, a_{20}, \\
a_{1}, a_{2}, a_{4}, a_{7}, a_{8}, a_{11}, a_{12} a_{20} \\
a_{2}, a_{4}, a_{8}, a_{9}, a_{12}, a_{20} \\
a_{1}, a_{2}, a_{3}, a_{4}, a_{5}, a_{6}, a_{7}, a_{8}, a_{9}, a_{12}, a_{16}, a_{20}\end{array}$ \\
\hline
\end{tabular}

Table 11: Asymmetric part of the possible preference relation: for each line, the car on the left is possibly preferred to the cars on the right, which, in turn, are not possibly preferred to the car on the left. For example, $a_{1}$ is possibly preferred to $a_{2}, a_{4}$ and $a_{8}$, while no one between $a_{2}, a_{4}$ and $a_{8}$ is possibly preferred to $a_{1}$.

\begin{tabular}{|c|c|}
\hline & Cars \\
\hline $\begin{array}{c}\left(a_{1}\right) \text { Audi A3 (3-doors) } \\
\left(a_{2}\right) \text { BMW } 1 \text { Series (3-doors) } \\
\left(a_{3}\right) \text { Hyunday ix20 } \\
\left(a_{4}\right) \text { Ford C-Max } \\
\left(a_{5}\right) \text { Toyota Aygo } \\
\left(a_{6}\right) \text { Seat Ibiza (5-doors) Style } \\
\left(a_{7}\right) \text { VolksWagen Polo highline 1.4 (3-door) } \\
\left(a_{8}\right) \text { BMW Serie 1 (3-doors) } \\
\left(a_{9}\right) \text { Chevrolet Spark } \\
\left(a_{10}\right) \text { FIAT Punto (3-doors) } \\
\left(a_{11}\right) \text { Ford Fiesta (3-doors) } \\
\left(a_{12}\right) \text { Honda Civic } \\
\left(a_{13}\right) \text { Kia Rio } \\
\left(a_{14}\right) \text { Lancia Ypsilon } \\
\left(a_{15}\right) \text { Mazda2 3-door Sporty } \\
\left(a_{16}\right) \text { Mercedes A-Class } \\
\left(a_{17}\right) \text { Mini Cooper } \\
\left(a_{18}\right) \text { Mitsubishi Space Star } \\
\left(a_{19}\right) \text { Nissan Micra } \\
\left(a_{20}\right) \text { Opel Corsa } \\
\left(a_{21}\right) \text { Peugeot 208 } \\
\left(a_{22}\right) \text { Renault Clio } \\
\left(a_{23}\right) \text { Skoda Citygo } \\
\left(a_{24}\right) \text { Suzuki Swift }\end{array}$ & $\begin{array}{c}a_{2}, a_{4}, a_{8}, \\
\emptyset \\
a_{1}, a_{2}, a_{4}, a_{5}, a_{6}, a_{7}, a_{8}, a_{12}, a_{20}, \\
a_{2}, \\
a_{1}, a_{2}, a_{4}, a_{6}, a_{7}, a_{8}, a_{12}, a_{20}, \\
a_{1}, a_{2}, a_{4}, a_{7}, a_{8}, a_{12}, a_{20}, \\
a_{1}, a_{2}, a_{4}, a_{8}, a_{12}, a_{20}, \\
a_{2}, \\
\emptyset \\
a_{1}, a_{2}, a_{3}, a_{4}, a_{5}, a_{6}, a_{7}, a_{8}, a_{9}, a_{11}, a_{12}, a_{14}, a_{15}, a_{16}, a_{19}, a_{20}, a_{21}, a_{22}, a_{22}, \\
a_{2}, a_{4}, a_{8}, a_{20}, \\
\emptyset \\
a_{1}, a_{2}, a_{4}, a_{5}, a_{6}, a_{7}, a_{8}, a_{9}, a_{11}, a_{12}, a_{15}, a_{16}, a_{19}, a_{20}, a_{21} \\
a_{1}, a_{2}, a_{4}, a_{6}, a_{7}, a_{8}, a_{9}, a_{11}, a_{12}, a_{15}, a_{16}, a_{20}, a_{21}, \\
a_{1}, a_{2}, a_{4}, a_{6}, a_{7}, a_{8}, a_{11}, a_{12}, a_{20}, \\
\emptyset \\
a_{1}, a_{2}, a_{4}, a_{8}, a_{12}, a_{16}, \\
a_{1}, a_{2}, a_{4}, a_{6}, a_{7}, a_{8}, a_{9}, a_{11}, a_{12}, a_{15}, a_{19}, a_{20}, a_{21}, \\
a_{1}, a_{2}, a_{4}, a_{6}, a_{7}, a_{8}, a_{9}, a_{11}, a_{12}, a_{20}, \\
\emptyset \\
a_{2}, a_{4}, a_{8}, a_{12}, a_{20}, \\
a_{1}, a_{2}, a_{4}, a_{7}, a_{8}, a_{12}, a_{20}, \\
a_{2}, a_{4}, a_{8}, a_{9}, a_{12}, a_{20}, \\
a_{2}, a_{3}, a_{4}, a_{5}, a_{6}, a_{7}, a_{8}, a_{9}, a_{11}, a_{16}, a_{20}\end{array}$ \\
\hline
\end{tabular}

To summarize the results of ROR, we build also the most representative model, being that one that maximally discriminate between alternatives for which there is a necessary preference. By computing 
the Choquet integral preference model of each alternative considering the most representative model, we obtain the following final ranking (in brackets the corresponding value obtained by applying the most representative model):

$$
\begin{aligned}
a_{24}(0.2415) \succ a_{10}(0.1520) & \succ a_{3}(0.135) \succ a_{14}(0.132) \succ a_{13}(0.1319) \succ a_{18}(0.1156) \succ a_{15}(0.1092) \succ \\
\succ a_{5}(0.1076) \succ a_{19}(0.1054) & \succ a_{21}(0.0992) \succ a_{6}(0.0984) \succ a_{22}(0.0971) \succ a_{23}(0.0858) \succ a_{7}(0.0813) \succ \\
\succ a_{11}(0.0707) \succ a_{17}(0.0614) & \succ a_{12}(0.0579) \succ a_{9}(0.0576) \succ a_{1}(0.0406) \succ a_{4}(0.0405) \succ a_{16}(0.0384) \succ \\
& \succ a_{8}(0.0355) \succ a_{2}(0.0354) \succ a_{20}(0.0096)
\end{aligned}
$$

From this representative ranking, we can state that $a_{24}$ is the best car, while the worst is surely $a_{20}$.

\section{Conclusions}

We considered multiple criteria decision aiding in case of interaction between criteria using the Choquet integral preference model. The application of the Choquet integral preference model requires that evaluations with respect to considered criteria are expressed on a common scale. We used AHP to build this common scale taking into account preference information given by the DM. To reduce considerably the number of pairwise comparisons usually required to the DM when applying AHP, we proposed to use AHP on a set of few reference points in the scale of each criterion and to interpolate the other values. Then, we adopted the recently introduced NAROR taking into account all the capacities compatible with the preference information provided by the DM. We illustrated the conjoint use of NAROR and AHP with an application to the decision problem of choosing a car to buy. We believe that the procedure we are proposing conjugates harmoniously the advantages of AHP in building a measurement scale and the advantages of the Choquet integral in handling interaction between criteria. In this context, the adoption of NAROR seems very beneficial because it permits to avoid focusing on only one capacity, which can be misleading for the reliability of the final decision. We believe also that the procedure of applying directly AHP on only a small set of reference points and using the linear interpolation to get the other values deserves to be considered generally in future applications, regardless from the use of the Choquet integral. Indeed, the high number of pairwise comparisons requested to the DM to apply AHP is a problem that can prevent its application in case of too many alternatives. Moreover, the request of too many pairwise comparisons can limit the reliability of the results supplied by AHP. In this perspective, our proposal can result very useful for application of AHP in all the many real life decision problems presenting a high number of alternatives. We conclude with an interesting extension of our method that we plan to develop in a future paper. It regards consideration of the probability that a given alternative has a certain rank or the probability that an alternative is preferred to another taking into consideration the whole set of compatible capacities according to the methodology proposed in [1].

\section{Acknowledgment}

The first two authors wish to acknowledge funding by the "Programma Operativo Nazionale" Ricerca \& Competitivitá "2007-2013" within the project "PON04a2 E SINERGREEN-RES-NOVAE". The authors would like to thank the four anonymous reviewers for their comments and suggestions which have helped to improve considerably this manuscript. 


\section{References}

[1] S. Angilella, S. Corrente, and S. Greco. Stochastic multiobjective acceptability analysis for the Choquet integral preference model and the scale construction problem. European Journal of Operational Research, 240:172-182, 2015.

[2] S. Angilella, S. Greco, F. Lamantia, and B. Matarazzo. Assessing non-additive utility for multicriteria decision aid. European Journal of Operational Research, 158(3):734-744, 2004.

[3] S. Angilella, S. Greco, and B. Matarazzo. The most representative utility function for nonadditive robust ordinal regression. In E. Hullermeier, R. Kruse, and F. Hoffmann, editors, Proceedings of IPMU 2010, LNAI 6178, pages 220-229. Springer, Heidelberg, 2010.

[4] S. Angilella, S. Greco, and B. Matarazzo. Non-additive robust ordinal regression: A multiple criteria decision model based on the Choquet integral. European Journal of Operational Research, 201(1):277-288, 2010.

[5] C. Bana e Costa and J.-C. Vansnick. MACBETH-An interactive path towards the construction of cardinal value functions. International transactions in operational Research, 1(4):489-500, 1994.

[6] C. Bana e Costa and J.-C. Vansnick. A critical analysis of the eigenvalue method used to derive priorities in AHP. European Journal of Operational Research, 187(3):1422-1428, 2008.

[7] D.E. Bell. Multiattribute utility functions: Decompositions using interpolation. Management Science, 25:744-753, 1979.

[8] L. Berrah and V. Clivillé. Towards an aggregation performance measurement system model in a supply chain context. Computers in Industry, 58(7):709-719, 2007.

[9] S. Bortot and R.A. Marques Pereira. Inconsistency and non-additive capacities: The Analytic Hierarchy Process in the framework of Choquet integration. Fuzzy Sets and Systems, 213:6-26, 2013.

[10] C. Boutilier, F. Bacchus, and R.I. Brafman. UCP-networks: A directed graphical representation of conditional utilities. In Proceedings of the Seventeenth conference on Uncertainty in artificial intelligence, pages 56-64. Morgan Kaufmann Publishers Inc., 2001.

[11] J.P. Brans and B. Mareschal. PROMETHEE Methods. In J. Figueira, S. Greco, and M. Ehrgott, editors, Multiple Criteria Decision Analysis: State of the Art Surveys, pages 163-196. Springer, Berlin, 2005.

[12] J.P. Brans and Ph. Vincke. A preference ranking organisation method: The PROMETHEE method for MCDM. Management Science, 31(6):647-656, 1985.

[13] A. Chateauneuf and J.Y. Jaffray. Some characterizations of lower probabilities and other monotone capacities through the use of Möbius inversion. Mathematical Social Sciences, 17:263-283, 1989.

[14] G. Choquet. Theory of capacities. Annales de l'institut Fourier, 5:131-295, 1954.

[15] S. Corrente, J.R. Figueira, and S. Greco. Dealing with interaction between bipolar multiple criteria preferences in PROMETHEE methods. Annals of Operations Research, 217(1):137-164, 2014. 
[16] S. Corrente, S. Greco, M. Kadziński, and R. Słowiński. Robust ordinal regression in preference learning and ranking. Machine Learning, 93:381-422, 2013.

[17] S. Corrente, S. Greco, M. Kadziński, and R. Słowiński. Robust ordinal regression. Wiley Enciclopedia of Operational Research, pages 1-10, 2014.

[18] M. Elliott. Selecting numerical scales for pairwise comparisons. Reliability Engineering and System Safety, 95(7):750-763, 2010.

[19] P.H. Farquhar. A Fractional Hypercube Decomposition Theorem for Multiattribute Utility Functions. Operations Research, 23:941-967, 1975.

[20] J. Figueira, S. Greco, and M. Ehrgott. Multiple Criteria Decision Analysis: State of the Art Surveys. Springer, Berlin, 2005.

[21] J. Figueira, S. Greco, B. Roy, and R. Słowiński. An overview of ELECTRE methods and their recent extensions. Journal of Multicriteria Decision Analysis, 20:61-85, 2013.

[22] J. Figueira, S. Greco, and R. Słowiński. Identifying the "most representative" value function among all compatible value functions in the GRIP. Proceedings of the 68th EURO Working Group on MCDA, Chania, October 2008.

[23] J. Figueira, V. Mousseau, and B. Roy. ELECTRE methods. Multiple Criteria Decision Analysis: State of the Art Surveys, pages 133-153, 2005.

[24] J.R. Figueira, S. Greco, and B. Roy. ELECTRE methods with interaction between criteria: An extension of the concordance index. European Journal of Operational Research, 199(2):478-495, 2009.

[25] P.C. Fishburn. Utility Theory for Decision Making. Wiley, New York, 1970.

[26] P.C. Fishburn and R.L. Keeney. Generalized utility independence and some implications. $O p$ erations Research, 23:928-940, 1975.

[27] A. Giarlotta and S. Greco. Necessary and possible preference structures. Journal of Mathematical Economics, 49(2):163-172, 2013.

[28] G. Gigerenzer and W. Gaissmaier. Heuristic decision making. Annual review of psychology, 62:451-482, 2011.

[29] I. Gilboa and D. Schmeidler. Additive representations of non-additive measures and the Choquet integral. Annals of Operations Research, 52:43-65, 1994.

[30] C. Gonzales and P. Perny. GAI networks for utility elicitation. In Proceedings of the Ninth International Conference on the Principles of Knowledge Representation and Reasoning, pages 223-234. CA: AAAI Press, 2004.

[31] M. Grabisch. The application of fuzzy integrals in multicriteria decision making. European Journal of Operational Research, 89:445-456, 1996.

[32] M. Grabisch. k-order additive discrete fuzzy measures and their representation. Fuzzy Sets and Systems, 92:167-189, 1997. 
[33] M. Grabisch, I. Kojadinovic, and P. Meyer. A review of methods for capacity identification in Choquet integral based multi-attribute utility theory: Applications of the Kappalab R package. European Journal of Operational Research, 186(2):766-785, 2008.

[34] M. Grabisch and C. Labreuche. Bi-capacities-Part I: definition, Möbius transform and interaction. Fuzzy Sets and Systems, 151(2):211-236, 2005.

[35] M. Grabisch and C. Labreuche. A decade of application of the Choquet and Sugeno integrals in multi-criteria decision aid. $4 O R, 6(1): 1-44,2008$.

[36] S. Greco, B. Matarazzo, and S. Giove. The Choquet integral with respect to a level dependent capacity. Fuzzy Sets and Systems, 175(1):1-35, 2011.

[37] S. Greco, V. Mousseau, and R. Słowiński. Ordinal regression revisited: multiple criteria ranking using a set of additive value functions. European Journal of Operational Research, 191(2):416436, 2008.

[38] S. Greco, V. Mousseau, and R. Słowiński. Robust ordinal regression for value functions handling interacting criteria. European Journal of Operational Research, 239(3):711-730, 2014.

[39] A. Ishizaka, D. Balkenborg, and T. Kaplan. Does AHP help us make a choice? An experimental evaluation. Journal of the Operational Research Society, 62(10):1801-1812, 2011.

[40] A. Ishizaka and A. Labib. Analystic Hierarchy Process and Expert Choice: benefits and limitations. OR Insight, 22(4):201-220, 2009.

[41] A. Ishizaka and M. Lusti. How to derive priorities in AHP: a comparative study. Central European Journal of Operations Research, 14(4):387-400, 2006.

[42] A. Ishizaka and P. Nemery. Multicriteria Decision Aid: Methods and software. Wiley, Chichester, 2013.

[43] A. Ishizaka and N.H. Nguyen. Calibrated fuzzy AHP for current bank account selection. Expert Systems with Applications, 40(9):3775-3783, 2013.

[44] R.L. Keeney. Analysis of preference dependencies among objectives. Operations Research, 29:1105-1120, 1981.

[45] R.L. Keeney and H. Raiffa. Decisions with multiple objectives: Preferences and value tradeoffs. J. Wiley, New York, 1993.

[46] J.L. Marichal and M. Roubens. Determination of weights of interacting criteria from a reference set. European Journal of Operational Research, 124(3):641-650, 2000.

[47] G.A. Miller. The magical number seven, plus or minus two: some limits on our capacity for processing information. Psychological review, 63(2):81, 1956.

[48] I. Millet. The effectiveness of alternative preference elicitation methods in the Analytic Hierarchy Process. Journal of Multi-Criteria Decision Analysis, 6(1):41-51, 1997.

[49] V. Mousseau, J. Figueira, L. Dias, C. Gomes da Silva, and J. Climaco. Resolving inconsistencies among constraints on the parameters of an MCDA model. European Journal of Operational Research, 147(1):72-93, 2003. 
[50] S. Murofushi, T. Soneda. Techniques for reading fuzzy measures (III): interaction index. 9th Fuzzy Systems Symposium, Sapporo, Japan, pages 693-696, 1993.

[51] J. Pérez, J.L. Jimeno, and E. Mokotoff. Another potential shortcoming of AHP. Top, 14(1):99$111,2006$.

[52] E. Rokou and K. Kirytopoulos. A calibrated group decision process. Group Decision and Negotiation, 23(6):1369-1384, 2014.

[53] G.C. Rota. On the foundations of combinatorial theory I. Theory of Möbius functions. Wahrscheinlichkeitstheorie und Verwandte Gebiete, 2:340-368, 1964.

[54] B. Roy and D. Bouyssou. Aide multicritère à la décision: méthodes et cas. Economica, 1993.

[55] T. Saaty. A scaling method for priorities in hierarchical structures. Journal of Mathematical Psychology, 15(3):234-281, 1977.

[56] T. Saaty. The Analytic Hierarchy Process. New York, McGraw-Hill, 1980.

[57] T. Saaty. How to make a decision: the Analytic Hierarchy Process. European Journal of Operational Research, 48(1):9-26, 1990.

[58] T. Saaty. Decision Making with Dependence and Feedback: The Analytic Network Process. RWS Publications, Pittsburgh, 1996.

[59] T.L. Saaty and M.S. Ozdemir. Why the magic number seven plus or minus two. Mathematical and Computer Modelling, 38(3):233-244, 2003.

[60] A. Salo and R.P. Hämäläinen. On the measurement of preferences in the Analytic Hierarchy Process. Journal of Multi-Criteria Decision Analysis, 6(6):309-319, 1997.

[61] G. Shafer. A Mathematical Theory of Evidence. Princeton University Press, 1976.

[62] L.S. Shapley. A value for n-person games. In H. W. Kuhn and A. W. Tucker, editors, Contributions to the Theory of Games II, pages 307-317. Princeton University Press, Princeton, 1953.

[63] M. Sugeno. Theory of fuzzy integrals and its applications. Tokyo Institute of Technology, 1974.

[64] P.P. Wakker. Additive representations of preferences: A new foundation of decision analysis, volume 4. Springer, Berlin, 1989.

[65] Y.-M. Wang, K.-S. Chin, and Y. Luo. Aggregation of direct and indirect judgments in pairwise comparison matrices with a re-examination of the criticisms by Bana e Costa and Vansnick. Information Sciences, 179(3):329-337, 2009.

[66] J.-Z. Wu and Q. Zhang. 2-order additive fuzzy measure identification method based on diamond pairwise comparison and maximum entropy principle. Fuzzy Optimization and Decision Making, $9(4): 435-453,2010$. 